Published in AIChE J., e16617, https://doi.org/10.1002/aic.16617, 2019

\title{
Enhancement of Mass Transfer from Particles by Local Shear-Rate and Correlations with Application to Drug Dissolution
}

\author{
Yanxing Wang ${ }^{1}$, James G. Brasseur ${ }^{2,3, *}$ \\ ${ }^{1}$ School of Aerospace Engineering, Georgia Institute of Technology, \\ Atlanta, GA 30332, USA \\ ${ }^{2}$ Smead Aerospace Engineering Sciences, University of Colorado, Boulder, CO 80309, USA \\ ${ }^{3}$ Mechanical Engineering, Pennsylvania State University, University Park, PA 16802, USA \\ *corresponding author
}

We analyze hydrodynamic enhancement of mass (or heat) release rate from small spherical particles within fluid flows from local flow shear-rate, with application to drug dissolution. Combining asymptotic theories in the high/low shear Peclet number limits in Stokes flow with 205 carefully-developed computational experiments, we develop accurate correlations for shear enhancement of Sherwood/Nusselt number $(S h / N u)$ as a function of shear Peclet and Reynolds number $\left(S^{*}, \operatorname{Re}_{S}\right)$. The data spanned $S^{*}$ from 0 500 and $\operatorname{Re}_{S}$ from 0 - 10. In Stokes flow our correlations are highly accurate over the entire $S^{*}$ range, whereas for finite $\operatorname{Re}_{S}<1$ accuracy is good for $S^{*}$ up to a few thousand. Shear enhancement results from highly three-dimensional spiraling flow created by particle spin. We develop a model for particle slip velocity that is inserted into the Ranz/Marshall correlation ${ }^{1}$ to show that shear-rate enhancement strongly dominates convection, a result important to drug dissolution.

Keywords: Dissolution, drug dissolution, particle mass transfer, particle heat transfer, shear enhancement, convective enhancement.

\section{Introduction}

The general problem of heat and mass transfer from small particles transported within a fluid flow has broad technological applicability. Our application is the release of drug molecules from the surfaces of large collections of "small" drug particles (up to particle radius $R \sim 100-150 \mu \mathrm{m}$ ) moving within liquids in the gastro-intestinal tract ("in vivo") and within impeller-driven dissolution measurement devices ("in vitro"). ${ }^{2}$ Within the pharmaceutical sciences community, the rate of molecular drug transport from clouds of drug particles previously within capsules or tablets ("dissolution") is a central determinant in the rate of drug absorption in the intestines. Because low drug solubility is common (as defined by the "BCS drug classification system"3), dissolution is often a rate-limiting step in absorption. Correspondingly, mathematical models of dissolution rate are central to pharmacokinetics model predictions of "bioavailability" and "bioequivalence."

As discussed in recent papers by Wang, et al. ${ }^{4,5}$, at the core of the prediction of dissolution-rate from large numbers of small drug particles over wide ranges of particle diameters is the prediction of molecular flux from the individual particles within the ensemble. For particles sufficiently small that diffusion is the leading order mechanism, the flux is appropriately normalized as follows:

$$
S h \equiv \frac{N_{S}^{\prime \prime}}{D_{m}\left(C_{S}-C_{B}\right) / R}=\frac{R}{\delta} .
$$


$S h$ is the "Sherwood number," where $N_{S}^{\prime \prime}$ is the average flux of molecules from the particle surface (moles/mass per unit area and time), $C_{S}$ is drug concentration at the drug particle surface (typically approximated at the aqueous solubility), $D_{m}$ is the diffusivity of drug molecules in the solvent of choice, and $R$ is the average particle radius. $C_{B}$ is the "bulk" (average) concentration of drug molecules in the solvent which, for unbounded dissolution, is the concentration at infinity $\left(C_{\propto}\right)$.

Defining the "diffusion layer thickness" $\delta$ from Fick's (or Fourier's) law as $N_{S}^{\prime \prime}=D_{m}[\partial C / \partial n]_{S} \equiv D_{m}\left[C_{S}-C_{B}\right] / \delta$, (1) can be written equivalently as $S h=R / \delta$. It can be shown that $S h=1$ for a single spherical particle experiencing purely diffusion-driven dissolution in "sink" conditions (i.e., effectively within an infinite domain), implying $\delta=R{ }^{4,5}$ Effects that enhance the rate of release of molecules from the particle surface cause increases in Sherwood number $S h$ and reductions in diffusion-layer thickness $\delta$ (relative to $R$ ). These considerations apply equally for mass and heat transfer where, for heat release, concentration is replaced by temperature, mass diffusivity by thermal diffusivity and Sherwood number by Nusselt number.

Confinement of molecules by a container surrounding the particle impacts the surface gradient in concentration and therefore creates deviations in $S h$ from 1. Furthermore, hydrodynamic enhancements to diffusion-driven dissolution occur in the presence of fluid flow relative to the particle surface, where $S h=$ 1 is the leading term in the limit of zero particle radius. Thus, for small particles the normalized flux can be written

$$
S h=1+\Delta_{\text {confine }}+\Delta_{\text {hydro }},
$$

where $\Delta_{\text {confine }}$ represents the modification of normalized flux from confinement and $\Delta_{\text {hydro }}$ represents enhancement in particle Sherwood number from hydrodynamic influences.

Wang et al. ${ }^{4}$ derived a closed-form solution to $\Delta_{\text {confine }}$ for diffusion-dominated dissolution from a spherical particle in an impermeable spherical container using a quasi-steady-state approximation and compared with the exact solution to show that the "confinement effect" $\Delta_{\text {confine }}$ is well approximated by the model. $\Delta_{\text {confine }}$ is generally a small enhancement for typical in vitro drug dissolution conditions, but can become significant when the volume of solid particles is not small relative to the container volume, as might be the case for drug dissolution in vivo.

The hydrodynamic enhancements to dissolution rate, $\Delta_{\text {hydro }}$, are functions of the appropriate particle Peclet and Reynolds numbers defined at the particle location with particle radius as an appropriate length scale. The most widely recognized hydrodynamic enhancement is "convective" enhancement associated with the magnitude of the relative velocity, $\Delta U \equiv|\Delta \mathbf{u}|$, between a moving particle and the surrounding flow. This "slip velocity" carries drug molecules into the wake which enhances surface flux and, correspondingly, increases concentration gradient at the particle surface and reduces the thickness of the surface diffusion layer. The result is an enhancement to the Sherwood number, $\Delta_{\text {conv }}$.

Ranz \& Marshall ${ }^{1}$ designed a clever experiment to produce accurate data with which $\Delta_{\text {conv }}$ was empirically parameterized for spherical particles with fixed surface concentration as a function of Peclet and Reynolds numbers, each defined with the magnitude of the relative (slip) velocity $\Delta U$ and average particle radius $R$ :

$$
\Delta_{\text {conv }}=0.424 P e_{\Delta U}^{1 / 3} \operatorname{Re}_{\Delta U}^{0.17},
$$

where $P e_{\Delta U} \equiv(\Delta U) R / D_{m}$ and $R e_{\Delta U} \equiv(\Delta U) R / v$ ( $v$ is the fluid kinematic viscosity). Thus, convective enhancement scales as $[(\Delta U) R]^{1 / 2}$. (Ranz \& Marshall wrote their correlation equivalently in terms of $\operatorname{Re}_{\Delta U}$ 
and Schmidt number $\mathrm{Sc}=v / D_{m}$.) We show in Appendix $A$ that in the small particle limit, $\Delta U \sim R^{2}$, so that $\Delta_{\text {conv }} \sim R^{3 / 2}$ for small particles.

Whereas convective enhancement to heat and mass transfer from relative velocity (slip) between particle and flow is the most commonly addressed hydrodynamic effect, in the current study we analyze a different mechanism for hydrodynamic enhancement of dissolution that originates in the existence of fluid shear-rate at the location of the particle, $\Delta_{\text {shear }}$. In the section below entitled "Physical Mechanisms" we show that the enhancement in surface flux results from vorticity-induced spin of fluid particles that generates a three-dimensional velocity field local to the particle surface, increasing the effective contact surface area for molecular flux. Using carefully developed high-resolution computational fluid dynamics with mass dissolution from spherical particles in the presence of uniform shear-rate, we develop empirical relationships between the Sherwood number and shear-rate-based Peclet and Reynolds numbers with Dirichlet boundary conditions.

Based on the empirical correlations we develop here between normalized flux $(S h)$ and the shear Peclet and Reynolds numbers, we show in Appendix $A$ that, although both convective and shear-rate effects vanish in the limit of vanishing particle size, $\Delta_{\text {shear }} / \Delta_{\text {conv }} \sim R^{-0.5}$ so that shear-rate is both highly significant and is the dominant hydrodynamic enhancement to mass transfer in the small-particle dissolution limit. Furthermore, the small-particle limit is increasingly relevant given the current trend towards "micronization" of drug particles to enhance dissolution rates with low solubility drugs ${ }^{6}$.

In the next section we describe former theoretical studies with asymptotic limits useful in the development of our correlations, and other relevant modeling studies. We then summarize our numerical approach (details are in Appendix B) and subtleties and care taken in the data generation process. From our simulations, we describe the essential physical mechanisms that underlie enhancement of mass transfer from local shear-rate and quantification of normalized flux (Sherwood number), vs. shear Peclet and Reynolds numbers. We then describe the development of our correlations with detailed analyses of the quality of the fit. We close by assessing sensitivity to changes in shear Peclet and Reynolds numbers, with details in Appendix C. Appendix A describes our model for slip velocity from which we present an analysis of shear-rate vs. convective enhancement to particle mass flux.

\section{Theoretical Background}

Here we consider a fluid particle feely suspended in a uniform unbounded shear flow, the second term in a Taylor series expansion of velocity relative to a moving solid particle.

\section{Nondimensional Parameters}

As previously discussed, the surface flux of molecules from small spherical drug particles normalized in the small-particle diffusion-dominated limit (the Sherwood number) is given by Eq. 1. Dimensional analysis in the small-particle limit implies that Sherwood number is a function of the appropriate particle Reynolds and Peclet numbers: $S h=f(P e, \mathrm{Re})$. Whereas the appropriate length scale in the diffusiondominated limit is particle radius $R$, the appropriate velocity scale depends on the mechanism underlying dissolution enhancement. Whereas for convective enhancement the velocity scale is slip velocity $\Delta U$, for shear-rate enhancement an appropriate velocity scale is $S R$, where $\mathrm{S}$ is flow shear rate at the particle and $R$ is particle radius. Thus the particle shear Reynolds and Peclet numbers are given by: 


$$
\operatorname{Re}_{S} \equiv \frac{S R^{2}}{v}, S^{*} \equiv \frac{S R^{2}}{D_{m}},
$$

where $v$ and $D_{m}$ are the diffusivities of momentum and mass, respectively. Note that we choose the symbol $S^{*}$ for shear Peclet number $\left(S^{*} \equiv P e_{S}\right)$ to emphasize what turns out to be the leading order influence of shear rate on dissolution from small particles at small particle Reynold numbers. By changing mass diffusivity to thermal diffusivity and Sherwood number to Nusselt number, the analyses and correlations in the current paper are applicable to heat transfer.

As previously discussed, surface flux of molecules (or mass) $N_{S}^{\prime \prime}$ is appropriately normalized by $D_{m}\left(C_{S}-C_{B}\right) / R$ in the diffusion-driven small-particle limit (bulk concentration $C_{B}$ is zero in an unbounded fluid with zero concentration at infinity). A primary aim of the current study is to empirically determine the relationship $S h=\operatorname{Sh}\left(S^{*}, \operatorname{Re}_{S}\right)$ between normalized flux and the shear Peclet and Reynolds numbers for spherical drug particles within a simple unbounded shear flow with Dirichlet surface concentration and no-slip surface velocity boundary conditions. From our resulting correlations, $\Delta_{\text {shear }}$ in Eq. (1) is found from $\Delta_{\text {shear }}=S h-1$, where $S h$ is Sherwood number with shear-rate as the only hydrodynamic influence $\left(\Delta_{\text {conf }}=0\right.$ in unbounded flow).

\section{Former Analytical and Modeling Studies}

The earlier efforts to characterize heat and mass transfer from particles in unbounded shear flows were asymptotic theories in the Stokes flow limit, beginning with Frankel and Acrivos ${ }^{7}$ who derived the following relationship between Sherwood/Nusselt number and shear Peclet number $S^{*}$ in the limit $S^{*} \rightarrow 0$ at zero Reynolds number with constant unbounded linear shear rate:

$$
S h_{0}=1+\kappa S^{* 1 / 2},
$$

where the subscript 0 indicates $\operatorname{Re}_{S}=0$. Frankel and Acrivos ${ }^{7}$ derived the proportionality constant $\kappa=\kappa_{A}$ $=0.257$. As discussed below, Batchelor ${ }^{8}$ used a different approach to derive the same asymptotic dependence for $S h_{0}\left(S^{*}\right)$ but with a proportionality constant $\kappa=\kappa_{B}=0.286,10 \%$ higher than that in the Frankel/Acrivos result. We shall show that our results are consistent with the Batchelor coefficient.

To evaluate the infinite Peclet number limit for Stokes flow, Acrivos ${ }^{9}$ argued that a freely suspended sphere in the limit of infinite shear Peclet number must be surrounded by a region of closed streamlines across which mass/heat is transferred by pure diffusion/conduction. For this reason, $S h$ asymptotically approaches a constant as $S^{*} \rightarrow \infty$. Acrivos ${ }^{9}$ took advantage of the analogy between the sphere and cylinder in simple shear flow to solve the mass transfer problem with an approximate method that yielded the asymptotic Sherwood number $S h_{0_{\infty}} \approx 4.5$ in the limit $S^{*} \rightarrow \infty$ for Stokes flow, a value consistent with CFD simulations by Yang et al. ${ }^{10}$

Batchelor $^{8}$ followed these important works with an extensive analysis of the steady-state transfer rate of mass/heat from a spherical particle within fixed mean velocity gradient profiles to derive formulas for Sherwood/Nusselt number in pure strain and rotation, as well as in the limit $S^{*} \rightarrow 0$ for uniform shear flow in the zero Reynolds number limit using a mathematical approach fundamentally different from that of Frankel and Acrivos ${ }^{7}$. As mentioned above, Batchelor ${ }^{8}$ derived the same asymptotic form as Frankel and Acrivos $^{7}$ (Eq. 5) but with a different proportionality constant.

The 1968 and 1971 theoretical studies above established the basis for the future studies on this subject. Using an advanced interpolation method, Polyanin and Dil'man ${ }^{11}$ developed the following approximate 
correlation for shear-induced enhancement to the Sherwood number at $\mathrm{Re}_{\mathrm{S}}=0$ (Stokes flow) as an interpolation between the Frankel and Acrivos ${ }^{7} S^{*} \rightarrow 0$ result and the approximate finite $S^{*} \rightarrow \infty$ limit of Acrivos ${ }^{9}$ :

$$
S h_{0}=1+0.26 S^{* 1 / 2} /\left(1+0.057 S^{* 1 / 2}\right)
$$

For small Peclet numbers $S h_{0} \approx 1+0.26 S^{* 1 / 2}$, close to the Frankel and Acrivos ${ }^{7}$ formula at small $S^{*}$ but slightly higher than Acrivos ${ }^{9}$ in the limit $S^{*} \rightarrow \infty$.

Analytic solutions at finite Reynolds numbers are precluded by nonlinearity. Subramanian and Koch $^{12,13}$ proved that at small finite Reynolds numbers, flow inertia breaks symmetry and produces open streamlines over neutrally-buoyant freely-suspended spheres in simple shear flow. As a result, unlike inertia-less flow $\left(\operatorname{Re}_{S} \equiv 0\right.$ ) where Sherwood number is finite in the limit $S^{*} \rightarrow \infty, S h$ (eventually) increases without bound as $S^{*} \rightarrow \infty$, even in the presence of only small levels of inertia. Subramanian and Koch ${ }^{13}$ also showed the existence of streamlines that spiral towards the sphere around the axis of rotation and, taking into account the confinement of thermal gradients to thin thermal boundary layers at high $\operatorname{Re}_{S} S^{*}$, they derived the following correlation that they state is applicable in the asymptotic limits $\operatorname{Re}_{s} \ll 1$ and $\operatorname{Re}_{s} \gg 1 /\left(S^{*}\right)^{2 / 5}$ :

$$
S h=\left(0.325-0.126 \operatorname{Re}_{s}^{1 / 2}\right)\left(\operatorname{Re}_{s} S^{*}\right)^{1 / 3}+O(1) .
$$

The second inequality implies that Eq. (7) applies when $S^{*} \gg 1 / \operatorname{Re}_{s}^{5 / 2}$; thus, since $\operatorname{Re}_{s} \ll 1$ the Sherwood number must be extraordinarily high for applicability of Eq. (7), making it of minimal applicability to dissolution from small (drug) particles. Yang et al. ${ }^{10}$ provided additional analysis of the Subramanian and $\operatorname{Koch}^{12,13}$ high $\operatorname{Re}_{S} S^{*}$ thermal boundary layer theory, including additional visual presentations of spiraling streamline structure and the development of numerical simulations to evaluate and validate the theory. As mentioned above, their simulations at their highest Peclet number simulations are consistent with the Acrivos ${ }^{9}$ Stokes flow asymptotic estimate $S h \rightarrow \approx 4.5$ as $S^{*} \rightarrow \infty$. They suggest that the influence of Reynolds number on Sherwood number is minimal when $\operatorname{Re}_{S} \leqslant 0.1$ and significant when $\operatorname{Re}_{S} \geq 1$. We comment on this conclusion in this work.

Longest and Kleinstreuer ${ }^{14}$ numerically simulated heat/mass transfer from a spherical droplet in a bounded simple shear flow with zero concentration on the walls. Using simulations in the range $S^{*} \sim 0.006 \rightarrow 19.2$ and $\operatorname{Re}_{S} \sim 0.003 \rightarrow 8.0$, they report the following approximate correlation by curve fitting their computational data: $S h=1.0+0.386 \operatorname{Re}_{s}^{0.237}\left(S^{*}\right)^{0.333}$. However, this form cannot be correct since it implies that $S h=1$ in Stokes flow independent of shear-rate.

\section{Aims of the Current Study}

Analytical methods cannot predict $S h\left(S^{*}, R e_{S}\right)$ at finite $\operatorname{Re}_{S}$ or over the complete range of $S^{*}$ in Stokes flow. Our aim is to apply high-resolution numerical models to accurately predict dissolution from feelyrotating spherical particles in unbounded linear shear flow at zero and finite $\mathrm{Re}_{S}$. To increase accuracy and to overcome numerical complications in modeling the Stokes flow limit, we provide the exact Stokes-flow solution to the lattice-Boltzmann method to estimate $S h$ vs. $S^{*}$ at $\operatorname{Re}_{S}=0$. We use the Acrivos ${ }^{9}$ analytical estimate $S h \rightarrow 4.5$ to extend the correlation to the infinite $S^{*}$ limit. We combine computational data in the ranges $0.01 \lesssim S^{*} \leqslant 500,0 \leqslant \operatorname{Re}_{S} \leqslant 10$ with asymptotic theory to produce accurate correlations with specific application to drug dissolution. However, the results are more generally applicable to particulate flows with $\operatorname{Re}_{S}<10$. 


\section{The Numerical Model}

Page limitations forced the details of the lattice-Boltzmann (LB) numerical method applied in the current study to be relegated to Appendix B. Here we summarize key issues surrounding the numerical model, grid resolution and the basic numerical method.

We model dissolution from a freely suspended spherical particle in an unbounded flow with uniform shear-rate. As illustrated in Figure 1, we use a dual-grid lattice-Boltzmann CFD method to predict the flow field, concentration field and surface mass flux from a highly-resolved spherical particle with radius $R$ placed symmetrically on the central plane of a Couette flow generated by two parallel plates moving in opposite directions with speed $U$, creating a background shear-rate $S=U / H$ in the steady state. The sphere is allowed to freely spin from surface frictional stresses driven by local shear-induced vorticity. Drug molecules are modeled as a passive scalar concentration field with specified mass diffusivity $D_{m}$. The initial flow field is devoid of drug molecules and the concentration field evolves from molecules released into the fluid at the sphere surface according to Fick's first law and driven by a Dirichlet boundary condition on scalar concentration.

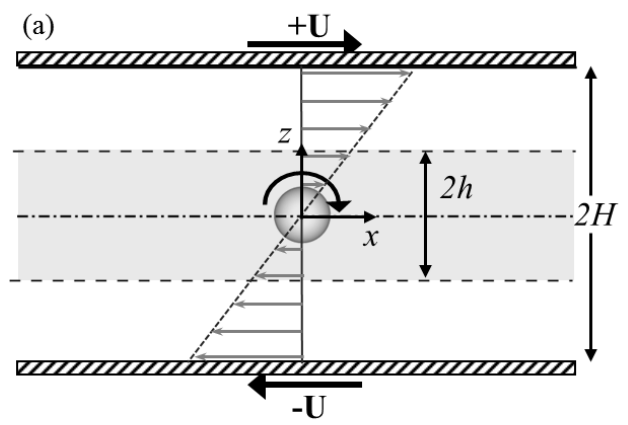

(b)

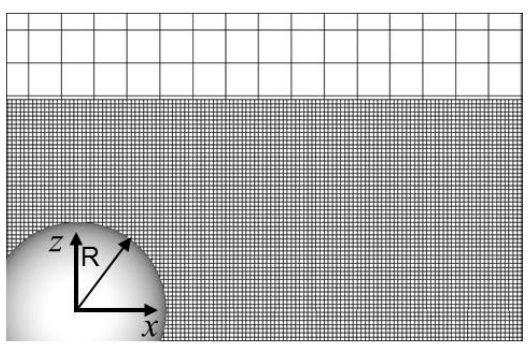

Figure 1. Physical model: (a) A spherical particle is suspended in a simple shear flow and resolved by a fine grid (grey area) of lateral dimension $2 h$ within a computational domain of size $2 L \times 2 H \times 2 W$ with $L / R=25, W / R=H / R=20$ or 40 . Shown is the $y=0$ plane; (b) the dual-grid (lattice) structure showing the high degree of resolution in the fine grid surrounding the particle. Details are discussed in the text.

No-slip boundary conditions are applied on all solid surfaces and periodic boundary conditions are applied at the streamwise and spanwise boundaries. To minimize the influence from these boundaries, the length, width and height of the computational domain $(2 \mathrm{~L}, 2 \mathrm{~W}, 2 \mathrm{H})$ is well over an order of magnitude larger than the sphere diameter (discussed below). Approximately 205 simulations were carried out to produce computational data over the range of shear Reynolds number $\operatorname{Re}_{S} \sim 0-10$ and shear Peclet numbers $S^{*} \sim 0.01$ - 500. This corresponds to Schmidt numbers $\left(S c=S^{*} / \operatorname{Re}_{S}\right)$ between 2 and 500 .

\section{The Lattice-Boltzmann Numerical Method}

Our LB model, mostly derived from advances by other researchers described in the literature (see references and details in Appendix B), has been used in several previous studies by Brasseur and colleagues with most details described and validated in Wang, et al. ${ }^{15}$. Briefly, our method is based on the D3Q15 discretization form of the Boltzmann equation in statistical physics with the dependent variable being a particle distribution function $f(\mathbf{x}, \xi, t)$ that quantifies the probability of an ensemble of fluid molecules at position $\mathbf{x}$ with velocity $\xi$ at time $t$. Continuum-level velocity $\mathbf{u}(\mathbf{x}, t)$ and density $\rho(\mathbf{x}, t)$ are obtained from moments of $f(\mathbf{x}, \xi, t)$ over velocity-space $\xi$. Distribution functions and macroscopic variables are 
represented on a discretized grid, or "lattice," and second order "bounce-back" boundary conditions are applied on boundary surfaces interpolated through the uniform cubical lattice cells. Furthermore, pressure does not appear explicitly in the Boltzmann equation and incompressible flow is modeled within a lowMach-number approximation with pressure made proportional to density. Compared with conventional boundary-fitted numerical methods for incompressible flow, the LB method handles complex geometries more easily and is more straightforward to parallelize due to the lack of a pressure term in the Boltzmann equation with corresponding local nature of interactions in physical space.

To evolve the scalar concentration over time within the flow field, we apply the "moment propagation method" with which the scalar concentration $\phi(\mathbf{x}, t)$ is propagated at the continuum level for each scalar using the discretized particle distribution function $f_{\alpha}(\mathbf{x}, t)$. Please see Appendix $B$ for references and for details.

\section{Resolution and the Dual Lattice}

In Appendix B we provide an analysis to assure adequate resolution of the velocity and concentration fields, and in the application of our dual-lattice approach. Please refer to the section "Resolution and the Dual Lattice" for details and references.

To minimize the influence of the boundary on the dissolution process, the relative distances to the boundaries of the computational domain to particle radius were maintained well over an order of magnitude (as large as possible within practical limits): $L / R=25, W / R=H / R=20$ (most cases) and 40 (5 lowest Reynolds number cases), as illustrated in Figure 1. Our aim was high-accuracy prediction of molecular release rate from a drug particle in an unbounded uniform shear flow. Special care was taken to minimize influences of confinement in the vertical on the flow and dissolution process. The domain was sufficiently long in the streamwise direction that steady state was reached before scalar released from the particle surface has reentered the computational domain. Since the concentration field never spread more than a couple diameters in the lateral direction, there was minimal influence of the lateral solid boundaries on the development and evolution of the scalar concentration field. Zero normal flux boundary conditions were applied on the lateral impermeable walls.

To reduce the computational load from an exceptionally fine uniform grid through the entire computational domain as required by the LB method to resolve exceptionally well a spherical particle in a local shear flow, we applied a dual-lattice method with a "fine grid" placed in a sub-region surrounding the particle, as shown in Figure $1 \mathrm{~b}$ with fine resolution of 44 lattice points over the particle diameter. The fine grid occupied a volume defined by $h / R$ between 2.1 and 3.6 according to the Reynolds number, with $h / R$ larger at the smallest Reynolds and Peclet numbers where diffusive effects are strongest. In the streamwise direction the fine grid extends to the domain boundaries, as illustrated in Figure 1. Outside this sub-region, the fine lattice couples to a lattice with resolution 6 - 10 times coarser than the fine lattice, depending on

Reynolds/Peclet number. We evolved the momentum field to steady state before turning on the release of scalar concentration from the particle surface and continued to the steady equilibrium state at which point dissolution rate is calculated at $S^{*}$ and $\operatorname{Re}_{S}$ controlled by specified shear rate, fluid kinematic viscosity and molecular diffusivity.

Please see Appendix $B$ for details and for an analysis of resolution. 


\section{Model for Zero Reynolds Number}

For the cases at $\operatorname{Re}_{S}=0$ (Stokes flow) with $S^{*}$ varying from zero to large values, we take advantage of the known analytical solution for velocity surrounding a spherical particle freely-suspended in an infinite simple shear flow ${ }^{16}$. To develop the most accurate Stokes flow correlation possible between $S h$ and $S^{*}$ within the LB framework, we assign the mass distribution functions values that produce the analytical solution (see Appendix B) on which the scalar concentration field evolves using the moment propagation method with fixed surface concentration. This approach both maximizes accuracy and greatly reduces computational cost.

\section{Physical Mechanisms for Enhancement of Scalar Flux}

We address basic mechanisms that underlie shear-induced enhancement in Figures 2 and 3. Figure 2 shows closely symmetric 3-D streamlines around a sphere at $\operatorname{Re}_{S}=0.1$ and $S^{*}=10$. Figure 2(a) shows streamlines initiated 3 radii upstream on an $x-y$ plane displaced in $z$ by $0.3 R$ that are associated with flow around the sphere outside the highly three-dimensional flow near the sphere surface shown in Figures 2 (b) and (c). Figures $2(\mathrm{~b}, \mathrm{c})$ show highly three-dimensional streamlines entering from the side along the $x$ axis driven by the spin of the particle at a rate proportional to the local shear rate. The 3D spiraling pattern in fluid particle trajectory near the surface forms a "rotation zone" region adjacent to the particle surface. At this finite Reynolds number, inertia causes the particles to move from the surface while mass conservation forces fluid from the sides of the particle to advect towards the sphere to fill the rotation zone. As a result, the streamlines in the rotation zone originate from the sides and spiral in towards the sphere and then out over the sphere surface. After leaving the rotation zone, the fluid particles combine with the outer nonrecirculating flow and travel downstream, a pattern consistent with Subramanian and Koch ${ }^{12,13}$ who argued

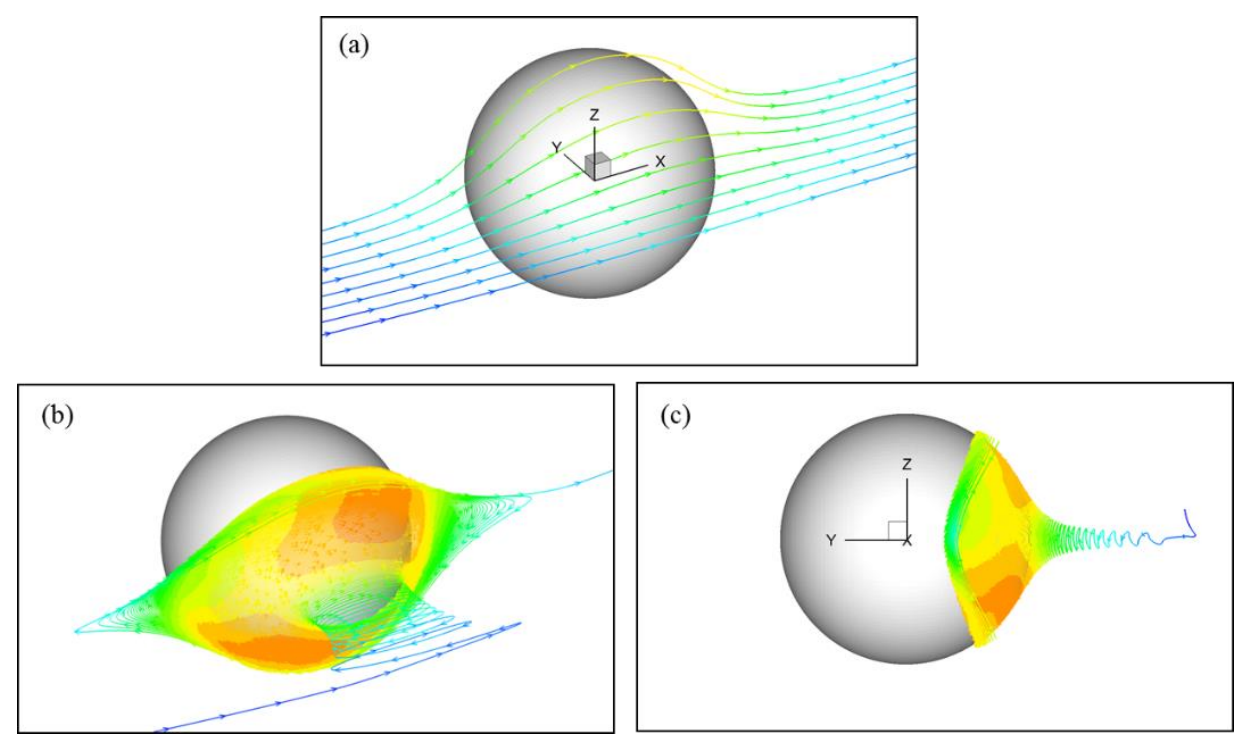

Figure 2. 3D streamline pattern around a spherical particle in a simple shear flow at $\operatorname{Re}_{S}=0.1, \quad S^{*}=10$. The color indicates scalar concentration with maximum and zero concentration given by red and blue, respectively. (a) Shows streamlines originating on an $x-y$ plane 3 radii upstream of the sphere displaced in $z$ by 0.3 radii; (b) and (c) show a streamline originating from a point transverse to the $x$ - $z$ plane that spirals around the rotation axis of the rotating sphere in helical fashion towards the sphere surface, continuing along the sphere surface to increasing effective surface area for mass transfer. 
that when Reynolds numbers is finite fluid particles in the rotation zone centrifuge out and destroy the closed streamline pattern that exists at $\operatorname{Re}_{S}=0$.

The highly 3D spiraling flow pattern exists also at zero Reynolds number, induced by the shear-driven spin of the particle, increasing the effective surface area for transport and enhancing dissolution rate. The three-dimensionality of the surface streamlines and the corresponding effective surface area and dissolution enhancement increase with spin-rate. Enhancement of mass flux implies increase in average surface concentration gradient and reduction in diffusion-layer thickness (Eq. 1, "Introduction"). Furthermore, increased three-dimensionality to the flow adjacent to the particle surface suggests increasing heterogeneity in local mass flux.

To clarify the topology of the flow around the sphere, in Figure 3 we plot the projected streamlines from the axial and vertical velocity components on streamwise-aligned vertical planes at different spanwise positions. The rotation zone around the sphere is connected horizontally to two recirculation zones separated by two lines of saddle points. Comparing different spanwise positions, the central rotation zone extends approximately one sphere radius in the spanwise direction at $\operatorname{Re}_{S} \ll 1$. At this low Reynolds number, a nearly closed stream surface exists round the sphere, according the theories of Acrivos ${ }^{9}$ and Subramanian and Koch. ${ }^{12,13}$ As $\operatorname{Re}_{S} \rightarrow 0$ the slight opening that allows streamlines to enter from the sides along the $\mathrm{x}$ axis (Figure 2) closes to form a closed stream surface across which transport is possible only through diffusion. At finite $\mathrm{Re}_{S}$ inertia introduces open streamlines and advective transport.

Figure 4 shows isocontours of scalar concentration on the central plane illustrating the advection of released with the shear flow. In Figures 4a,b $S^{*}$ changes from 10 to 100 with $\operatorname{Re}_{s}$ fixed at 0.1 while in
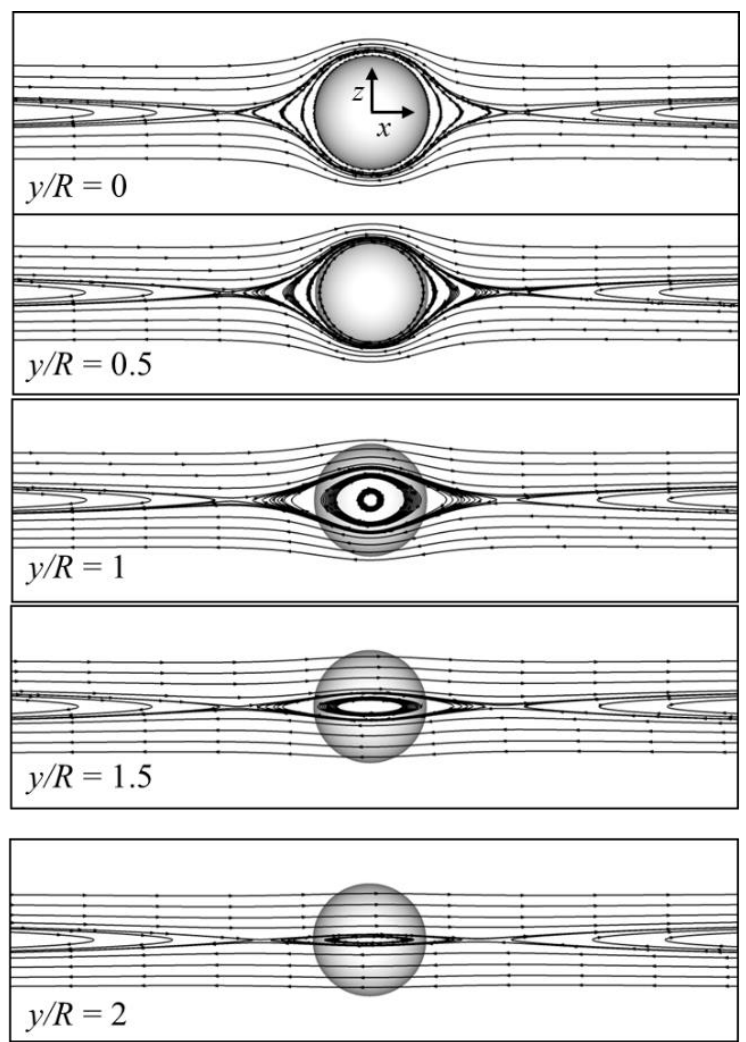

Figure 3. Projected streamlines on $x-z$ planes at different transverse positions. $\operatorname{Re}_{\mathrm{S}}=0.1, \mathrm{~S}^{*}=10$.

(a)

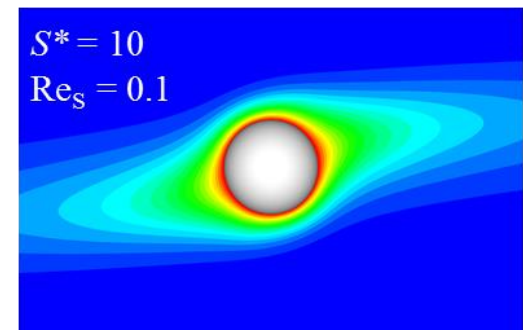

(b)

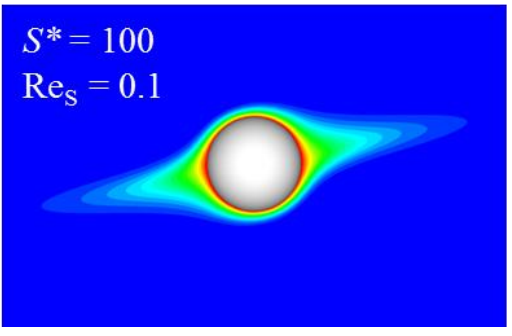

(c)

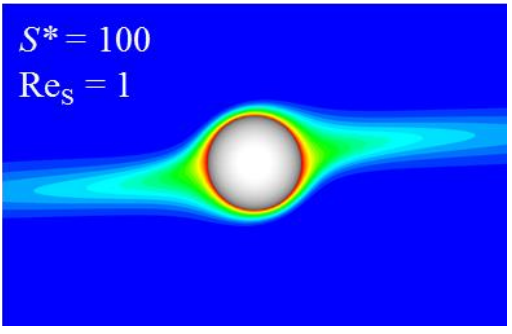

Figure 4. Patterns of scalar concentration around a spherical particle in a simple shear flow. $S^{*}$ is varied from (a) to (b) at fixed $\operatorname{Re}_{S}=0.1$ and $\mathrm{Re}_{\mathrm{S}}$ is varied from (b) to (c) with fixed $\mathrm{S}^{*}=100$. Concentration is maximum at the particle surface (dark red) and zero far from the sphere (dark blue). Note that the computational domain is much larger than shown. 
Figures $4 \mathrm{~b}, \mathrm{c} \operatorname{Re}_{S}$ changes from 0.1 to 1.0 with $S^{*}$ fixed at 100 . At fixed Reynolds number, higher $S^{*}$ corresponds to higher shear rate or lower diffusivity. Figures $4 \mathrm{a}, \mathrm{b}$ show that the effective reduction in diffusivity leads to sharper, or less smooth, scalar distributions with possible cusp formation at an angle rotated from the principle strain direction $\left(45^{\circ}\right)$ by vorticity towards the $x$ axis. We anticipate sharper cusps with higher $S^{*}$, implying larger variations in local surface flux over the surface of the particle with increasing $S^{*}$. Comparing Figures $5 \mathrm{~b}$ and 5 c suggests that increasing inertia $\left(\operatorname{Re}_{S}\right)$ may interfere with cusp formation, perhaps also reducing the highest gradients in surface heat flux. We observe that as $\operatorname{Re}_{S}$ increases from 0.1 (b) to 1.0 (c), at fixed $S^{*}=100$, the isocontour pattern smooths somewhat, conceptually consistent with the corresponding reduction in Schmidt number with increasing $\operatorname{Re}_{S}$ (at fixed $S^{*}$ ).

Consider mass release and transport of molecules from particles within a flow with fixed fluid properties: fluid kinematic viscosity and scalar diffusivity. Then higher vs. lower local particle $S^{*}$ (at fixed $\operatorname{Re}_{S}$ ), or higher vs. lower $\operatorname{Re}_{S}$ (at fixed $S^{*}$ ), can be interpreted as higher or lower flow shear-rate at the location of the particle. As a result, as local particle $S^{*}$ and $\operatorname{Re}_{S}$ increase, so do local particle flow strainrate, vorticity magnitudes and particle spin-rate. In context with the discussion surrounding Figures 2 and 3 , then, increases in particle $S^{*}$ or $\operatorname{Re}_{S}$ correlate with the enhancement of net mass flux and higher Sherwood number. However, to assess the relative sensitivities in anticipated increases in $S h$ relative to increases in local particle $S^{*}$ vs. $\operatorname{Re}_{S}$ requires quantification, the primary aim in what follows. An objective in the current study is the development of practical correlations, $\operatorname{Sh}\left(S^{*}, \operatorname{Re}_{S}\right)$, for dissolution from spherical drug particles in the presence hydrodynamic shear-rate over variations in $S^{*}$ and $\operatorname{Re}_{S}$ likely experienced by drug particle dissolution in the human intestinal track (in vivo) as well as within in vitro devices commonly used for product comparison. ${ }^{3,17}$ The final empirical relationships developed in this study will find applicability over a wide range of dissolution and heat-transfer processes where concentration or temperature are fixed and known on the particle surface.

\section{Quantitative Assessment of the Shear-rate Enhancement of Scalar Flux}

We created 205 simulations and data points in steady state with specified $S^{*}$ and Res. From each simulation, we calculated the surface flux and Sherwood number $S h$ (Eq. (1) with $C_{B}=0$ ). In Figure 5 we plot $S h$ against $S^{*}$ at fixed $\operatorname{Re}_{S}$ (Figure 5a,b) and we plot $S h$ against $\operatorname{Re}_{S}$ at fixed $S^{*}$, in linear and $\log$ scales (Figure 5c,d). Shear Reynolds number $\operatorname{Re}_{S}$ varies from 0 (the Stokes flow limit) to 10 and shear Peclet number $S^{*}$ from 0 to 500. Numerical instability limited the lowest Reynolds number range $\operatorname{Re}_{S} \lesssim 0.1$ to $S^{*}<10$.

The pure diffusion limit in an infinite domain is given by $S h=1 .{ }^{4,5}$ Figure 5 shows that the enhancement of dissolution from hydrodynamic shear-rate is, quantitatively, a strong effect. A factor of two increase in surface flux from the diffusion limit occurs at quite low $S^{*}(\approx 10)$, with additional enhancement associated with increasing $\operatorname{Re}_{S}$ (i.e., reducing Schmidt number). However, the sensitivity in the response of $S h$ to $S^{*}$ vs. $\operatorname{Re}_{S}$ is quite different. Figures $5(\mathrm{~d})$ and $5(\mathrm{~b})$ indicate that when $S^{*} \lesssim 10$ there is very little dependence on Reynolds number, (up to $\operatorname{Re}_{S} \approx 10$ ) while at higher shear Peclet numbers, $S^{*} \geq 100-200$, Reynolds number $\sim 1-10$ can lead to additional increases in $S h$ from the Stokes flow value from roughly 3 to roughly $4(\sim 25 \%)$.

From Figure 5(a) we learn that the rate of enhancement of dissolution rate is rapid in the transition from pure diffusion to small levels of flow shear-rate $\left(S^{*} \lesssim 10\right)$. However, with further increases in shear-rate ( $S^{*}$ exceeding $\sim 10-100$ ) the rate of growth in $S h$ slows considerably. The finite Acrivos ${ }^{9}$ limit $\mathrm{Sh}$ $S h \rightarrow \approx 4.5$ as $S^{*} \rightarrow \infty$ in Stokes flow is qualitatively consistent with the black $\operatorname{Re}_{S}=0$ curve in Figure 
5(a). As previously discussed, flow inertia breaks the closed streamlines around the sphere ${ }^{12,13}$ with the consequence that $S h$ increases without bound in the limit $S^{*} \rightarrow \infty$ at finite $\operatorname{Re}_{S}$. Figure 5 suggests that the rate of growth in the high $S^{*}$ limit increases with increasing $\operatorname{Re}_{S}$.

Yang et al. ${ }^{10}$ suggest that the influence of Reynolds number on the Sherwood number is weak when $\operatorname{Re}_{S}<0.1$. Figure 5 indicates that, whereas this may be the case when $S^{*} \leqslant 10-100$, when $S^{*}$ exceeds 100 or so there exists significant enhancement of $S h$ above the Stokes flow value even at $\operatorname{Re}_{S} \approx 0.1$. This additional inertia-induced enhancement increases with $S^{*}$ more rapidly with increasing $\operatorname{Re}_{S}>0$. However, Figure $5(\mathrm{~b}, \mathrm{c})$ shows that at fixed $S^{*}$ the rate of increase in $\mathrm{Sh}$ with increasing $\operatorname{Re}_{S}$ is negligible when $S^{*}<10$ or so, and modest at higher $S^{*}$, up to $S^{*} \approx 500$.

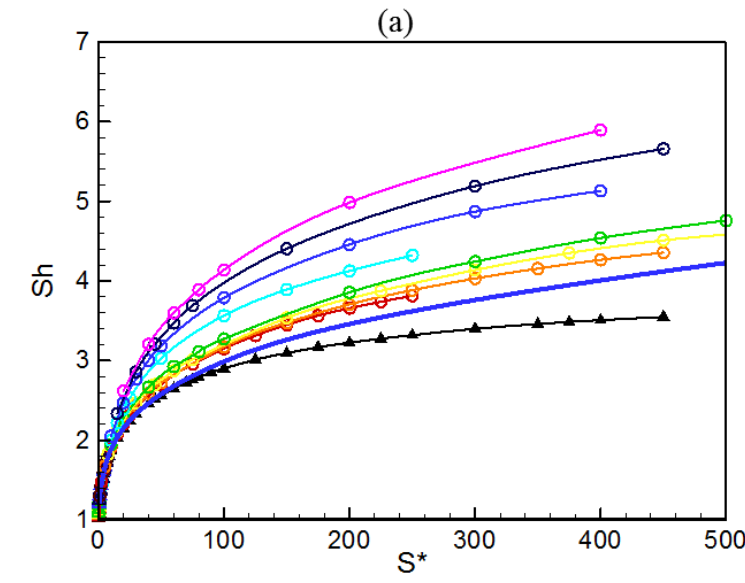

(c)

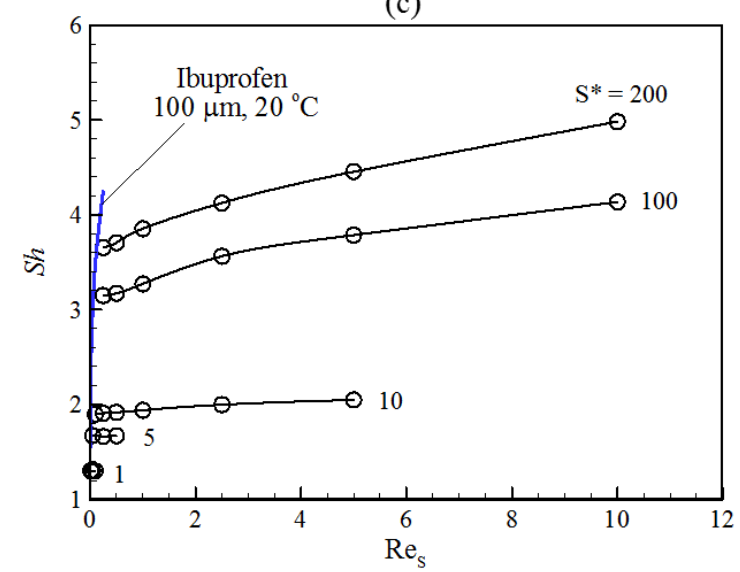

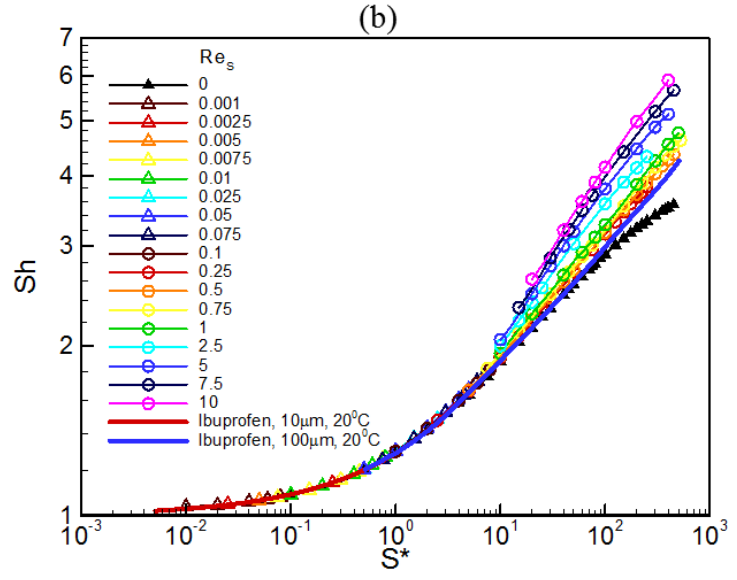

(d)

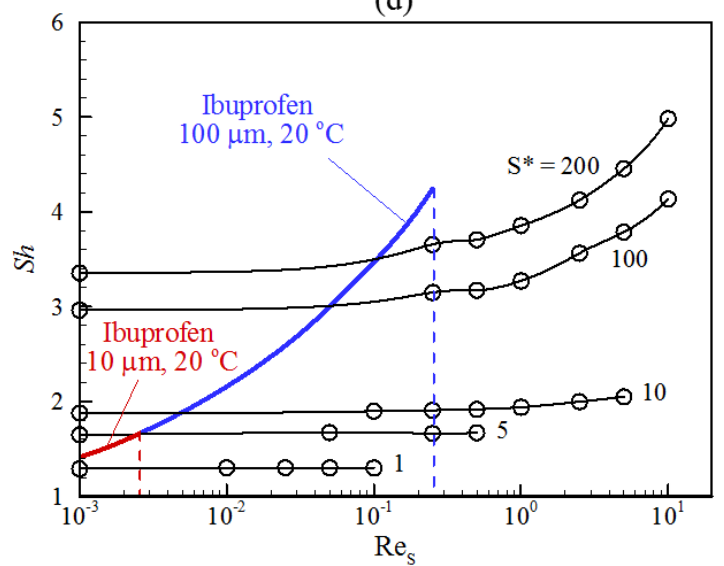

Figure 5. Variation of normalized surface flux $(S h)$ with shear Peclet $\left(S^{*}\right)$ and Reynolds $\left(\operatorname{Re}_{S}\right)$ numbers, numerical simulation, with linear and $\log$ scales. (a,b): $S h$ versus $S^{*}$ at fixed $\operatorname{Re}_{S}$; .(c,d): $S h$ versus $\operatorname{Re}_{S}$ at fixed $S^{*}$. The heavy solid red and blue curves in Figures (a), (b) and (d) show the variation in $S^{*}, \operatorname{Re}_{S}$ and $S h$ for dissolution of an ibuprofen drug particle of diameter $10 \mu \mathrm{m}$ (red) and $100 \mu \mathrm{m}$ (blue) in water at 20C with shear-rate variation from 0 to $100 \mathrm{~s}^{-1}$.

Consider dissolution from typical drug particles. Specifically, consider the dissolution of $10 \mu \mathrm{m}$ and $100 \mu \mathrm{m}$ ibuprofen drug particles in water at 20C, a common drug and typical size drug particle sizes during dissolution. As the drug particles move within the intestines, they experience variations in shear-rate up to 10-20 $\mathrm{s}^{-1}$ (Appendix A, based on Banco ${ }^{18}$ ). In in vitro testing devices, commonly required for FDA 
certification, local shear-rates can easily exceed $100 \mathrm{~s} \mathrm{~s}^{-1} \cdot{ }^{17}$ In Figures 5(a) - (d) there are solid red and blue curves that show the variations in $S^{*}, \mathrm{Re}_{S}$ and $S h$ for ibuprofen drug particles of diameter $10 \mu \mathrm{m}$ (red) and $100 \mu \mathrm{m}$ (blue) in water at $20 \mathrm{C}$ with shear-rate variation between 0 and $100 \mathrm{~s}^{-1}$. $\operatorname{Re}_{S}$ never exceeds 0.25 (Figure 5(d)) and the impact of shear Peclet number on the variation of $S h$ is a great deal stronger than that of Reynolds number. Indeed, for drug dissolution in vivo, the impact of $\operatorname{Re}_{S}$ is negligible and a correlation for the dependence of $S h$ on $S^{*}$ in Stokes flow is sufficient for typical pharmaceutical applications.

To validate the numerical methods and evaluate the accuracy of the numerical results, we compare in Figure 6 our results with those available in the literature as discussed in "Former Analytical and Modeling Studies" above. Using very different asymptotic methods, Frankel and Acrivos ${ }^{7}$ and Batchelor ${ }^{8}$ analytically derived the asymptotic dependence of Sherwood number for Stokes flow to scale on $\sqrt{S^{*}}$ in the limit

Figure 6. Comparisons of our computational data in Stokes flow with the asymptotic theories of Frankel/Acrivos ${ }^{7}$ and Batchelor ${ }^{8}$ for $S^{*} \rightarrow 0$ (Eq. 5) and the Polyanin and Dil'man ${ }^{11}$ interpolation between the Frankel/Acrivos $S^{*} \rightarrow 0$ limit and the Acrivos $^{9}$ approximate limit $S h \rightarrow 4.5$ as $S^{*} \rightarrow \infty$.

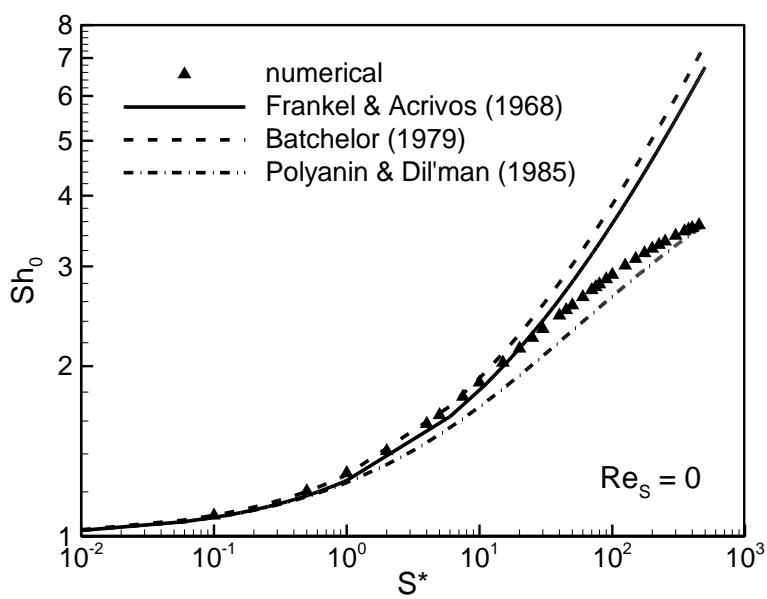

$S^{*} \rightarrow 0$, with the two slightly different constants of proportionality (see "Former Analytical and Modeling Studies" and Eq. 5). Polyanin and Dil'man ${ }^{11}$ developed Eq. (7), an interpolation between the Frankel and Acrivos $^{7} S^{*} \rightarrow 0$ limit and the approximate limit finite $S h \rightarrow 4.5$ as $S^{*} \rightarrow \infty$ of Acrivos ${ }^{9}$ at $\operatorname{Re}_{S}=0$. Figure 6 shows that our data compare well with the three expressions in the limit $S^{*} \rightarrow 0$, however the Polyanin and Dil'man ${ }^{11}$ interpolation does not compare well with our computational data at intermediate shear Peclet numbers. We find that Batchelor's proportionality constant in the expression derived by Frankel and Acrivos $^{7}$ and Batchelor ${ }^{8}$ for the $S^{*} \rightarrow 0$ limit is a better fit to our data than the constant derived by Frankel and Acrivos $^{7}$ (i.e., Eq. 5 with $\kappa=\kappa_{B}$ ).

We do not compare Eq. (7) with our data because, as pointed out previously, the asymptotic limits under which the expression was derived do not apply to the parameter ranges for which our computational data were collected. We also do not show our comparisons with the expression presented by Longest and Kleinstreuer ${ }^{14}$ because, as pointed out previously, the expression is fundamentally incorrect in the Stokes flow limit. Consequently, the expression compares poorly over all with our data, both qualitatively and quantitatively.

Clearly there is a need for accurate correlations that can be applied to mass and heat release from small particles (such as drug particles), particularly at finite shear Reynolds numbers and Peclet numbers far from the zero Peclet number theoretical limit. Reynolds and Peclet numbers far more common in practice. In the following sections, we use the asymptotic theories of Frankel and Acrivos ${ }^{7}$ and Acrivos $^{9}$ to guide the development of correlations using our 205 carefully developed simulations as described above. 


\section{Development of the Correlation at Zero Reynolds Number}

Given the dominance of the $S^{*}$ in the determination of shear enhancement of dissolution rate, our strategy to develop a useful accurate empirical correlation for $\operatorname{Sh}\left(S^{*}, \operatorname{Re}_{S}\right)$ is to first develop the correlation $S h_{0}\left(S^{*}\right)=S h\left(S^{*}, 0\right)$ for Stokes flow followed by development for Reynolds-number-dependent deviations from Stokes flow, $S h-S h_{0}$. Here we develop the correlation for $S h_{0}$

The variation of $S h_{0}$ with $S^{*}$ is too complex to represent accurately with a single mathematical expression over all $S^{*}$. We use the asymptotic theories (Eq. 5) to argue for square root dependence $S h_{0} \sim S^{* 1 / 2}$ in the limit $S^{*} \rightarrow 0$ and the Acrivos ${ }^{9}$ theory to argue for the limit $S h \rightarrow 4.5$ as $S^{*} \rightarrow \infty$. Thus, we search for three ranges in $S^{*}$ with power-law dependence.

In Figure 7(a) we plot $S h_{0}-1$ and $4.5-S h_{0}$ against $S^{*}$ on $\log -\log$ scales. These curves show power law dependence in the regimes $S^{*} \leqslant 5$ and $S^{*} \geq 100$. Figure $7 \mathrm{~b}$, a log-log plot of $S h_{0}$ vs. $S^{*}$ in the range $S^{*}$ $\sim 0.1$ to 500, indicates that a power law is also a good approximation in the intermediate range $5 \leq S^{*} \leq 100$. Standard linear least squares fit of $\ln \left(S h_{0}-1\right)$ vs. $\ln \left(S^{*}\right)$ in the range $S^{*} \leq 5, \ln \left(S h_{0}\right)$ vs. $\ln \left(S^{*}\right)$ in the range $5 \leq S^{*} \leq 100$ and $\ln \left(4.5-S h_{0}\right)$ vs. $\ln \left(S^{*}\right)$ in the range $S^{*} \geq 100$ produces the following:

$$
\begin{array}{ll}
S h_{0}=1+0.290\left(S^{*}\right)^{0.504} & 0.1 \leq S^{*}<5 \\
S h_{0}=1.206\left(S^{*}\right)^{0.192} & 5 \leq S^{*}<100 \\
S h_{0}=4.5-8.064\left(S^{*}\right)^{-0.349} & 100 \leq S^{*}<500
\end{array}
$$
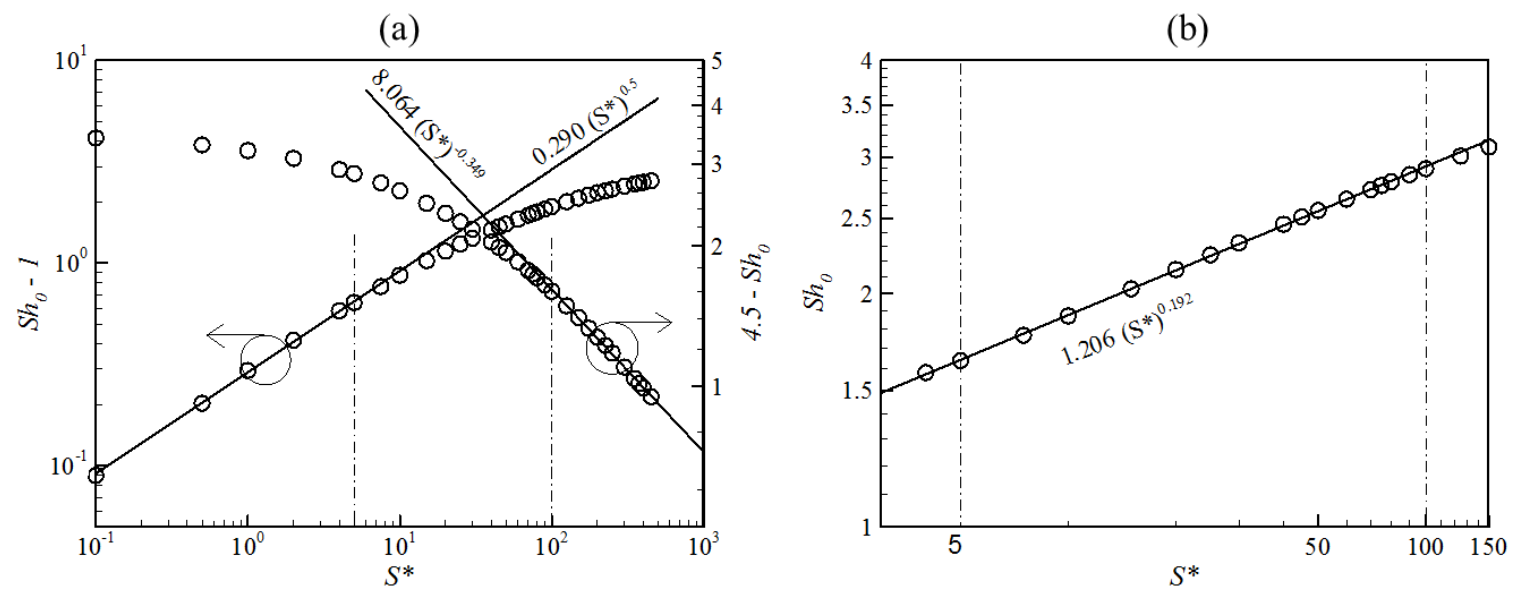

Figure 7. Least square fitting based on power laws at $\operatorname{Re}_{S}=0$. (a) $S h-1$ vs. $S^{*}$ for $S^{*} \leq 5$ and $4.5-S h$ vs. $S^{*}$ for $S^{*} \geq 100$; (b) $S h$ vs. $S^{*}$ for $5 \leq S^{*} \leq 100$.

The low $S^{*}$ correlation is very close to the $\sqrt{S^{*}}$ dependence predicted theoretically in the asymptotic limit. For consistency with this theoretical limit, we adjust the power on $S^{*}$ slightly from 0.504 to 0.500 . In addition, because we developed three fits independently in each of the three $S^{*}$ ranges, the curves are not precisely continuous at the boundaries between the regimes, $S^{*}=5$ and $S^{*}=100$. To force continuity at 
these two boundaries, we adjust the coefficient and power of the intermediate regime correlation slightly to produce our final correlations for $S h_{0}$ :

$$
\begin{array}{lll} 
& S h_{0}=1+0.290\left(S^{*}\right)^{0.5} & S^{*} \leq 5 \\
\text { final } S h_{0} \text { correlation: } & S h_{0}=1.219\left(S^{*}\right)^{0.187} & 5 \leq S^{*} \leq 100 \\
& S h_{0}=4.5-8.064\left(S^{*}\right)^{-0.349} & S^{*} \geq 100
\end{array}
$$

The asymptotic theories of Frankel/Acrivos ${ }^{7}$ and Batchelor ${ }^{8}$ produce the same $S h_{0}-1=\kappa S^{* 1 / 2}$ prediction (Eq. 5), but with constants $\kappa$ that differ by about $10 \%\left(\kappa_{A}=0.257, \kappa_{\mathrm{B}}=0.286\right)$. The constant we developed empirically $(0.290)$ is much closer to the Batchelor constant consistent with the observation from Figure 6, indicating support for the Batchelor result.

The $S h_{0}$ correlations above are compared with the computational data at $\operatorname{Re}_{S}=0$ in Figure 8 with both linear and log scales. Over the range of the data, the comparisons are excellent. Given that these correlations were determined using high-accuracy computational data over 3.5 orders magnitude change in $S^{*}$ (from 0.1 to 500) combined with an accurate asymptotic form in the zero $S^{*}$ limit together with a reasonable analytical estimate for the infinite $S^{*}$ limit, we argue that these correlations are reasonable over the entire range of shear Peclet numbers.

In the low $S^{*}$ regime, our results differ from $\sqrt{S^{*}}$ dependence in the $S^{*} \rightarrow 0$ limit by only $0.5 \%$ at $S^{*}$ $=5$ suggesting a reasonable $S^{*}$ range of validity of the $\sqrt{S^{*}}$ dependence based on our data. Figure 8 indicates that the correlations in Eq. (19) are accurate up to $S^{*} \approx 500$ where a power law

fit our data well (Figure 7). We extend the power law behavior observed between $S^{*}$ of 100 and 500 to infinity using the Acrivos ${ }^{9}$ estimate of 4.5 for the asymptotic limit. The relative difference between $S h_{0}$ at $S^{*}=500$ and $S^{*}$ at infinity is only $20 \%$, suggesting that the extension of the power law from $S^{*}=500$ to 4.5 at $S^{*}=\infty$ is quite reasonable and produces estimates for $S h$ at high $S^{*}$ that are as accurate as the Acrivos estimate for the asymptotic limit.

(a)

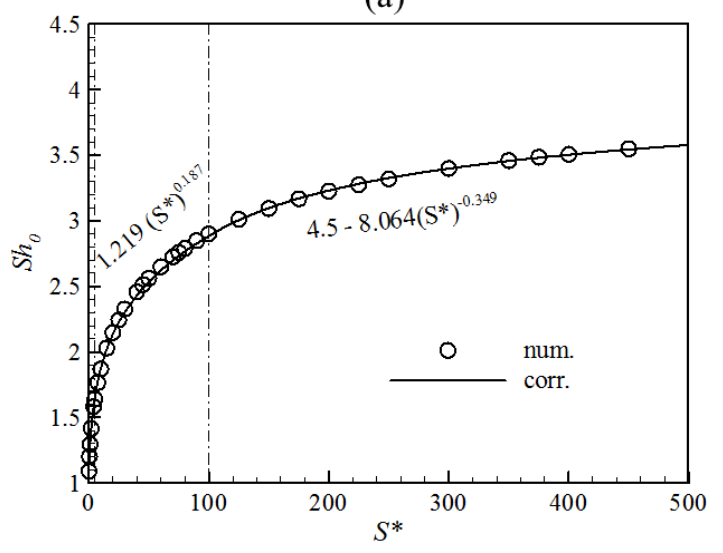

(b)

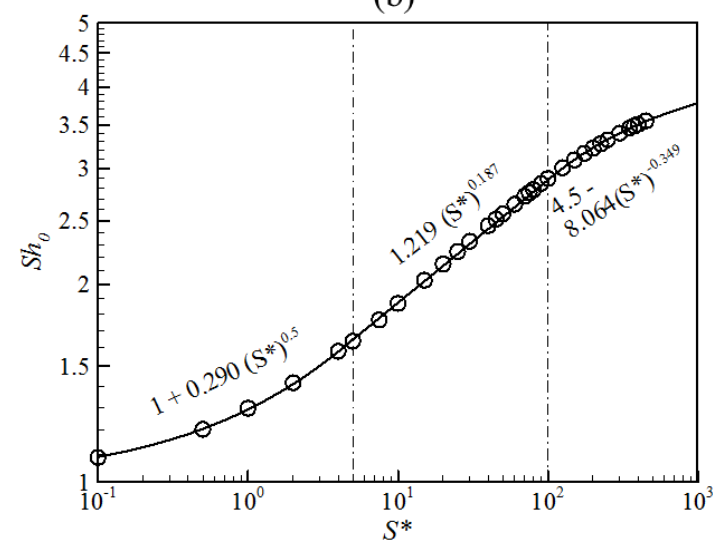

Figure 8. The comparison of correlations in Eqs. (19) with the computational data obtained at $\operatorname{Re}_{S}=0$. (a) linear and (b) logarithmic scales are shown. 


\section{Correlations for Non-zero Reynolds Numbers}

To develop the correlations for non-zero Reynolds numbers, we search for deviations from the zero Reynolds number correlations in Eq. (9) such that the finite Reynolds number correlations reduce to those at $\operatorname{Re}_{S}=0$ as $\operatorname{Re}_{S}$ decreases to 0 . We anticipate, therefore, a degradation in confidence, compared to our correlations for $S h_{0}$, at higher Reynolds numbers. This will be particularly the case at higher $S^{*}$, for two reasons. Firstly because it is proven that $S h$ increases indefinitely as $S^{*} \rightarrow \infty$ with unknown asymptotic form $^{12,13}$. Secondly, because high Peclet numbers are accompanied by high Schmidt numbers which lead to numerical instability. Therefore, the highest $S^{*}$ range over which our correlations can be reasonably extrapolated outside the parameter range of the data upon which our correlations are based $\left(0<S^{*} \leq 500,0 \leq \operatorname{Re}_{S}<10\right)$ degrades at Reynolds numbers "far" from Stokes flow, likely Reynolds numbers exceeding $\sim O(1)$.

Given the lack of asymptotic theory upon which to anchor correlations at finite $\operatorname{Re}_{S}$, and given the increasing sparseness of the computational data in the $S^{*}-\operatorname{Re}_{S}$ parameter space at higher $\operatorname{Re}_{S}$ (see Figure 5), we search for a single empirical relationship for $S h$ - $S h_{0}$, initially with a form consistent with the RanzMarshall ${ }^{1}$ correlation for estimation of Sherwood (or Nusselt) number for convective enhancement of mass (or heat) transfer from spherical particles in the small-particle limit. This correlation, shown in Eq. (3), has the following form:

$$
S h=S h_{0}+e^{a}\left(S^{*}\right)^{b}\left(\operatorname{Re}_{S}\right)^{c}
$$

equivalent to the following linear logarithmic form:

$$
\ln \left(S h-S h_{0}\right)=a+b \ln (S *)+c \ln \left(\operatorname{Re}_{S}\right) .
$$

We use the data at fixed values of $\operatorname{Re}_{S}$ to estimate the model coefficients $a, b$ and $c$ using standard multivariable linear least squares regression on Eq. (11) with our computational data. These coefficients are listed under "correlation 1" in Table 1.

Table 1. Coefficients of correlations 1 and 2

\begin{tabular}{l|c|c|c|c}
\hline & $a$ & $b$ & $c$ & $d$ \\
\hline correlation 1 & -4.036 & 0.684 & 0.460 & \\
\hline correlation 2 & -3.977 & 0.674 & 0.583 & -0.032 \\
\hline
\end{tabular}

The resulting correlation is plotted in Figure 9 (a) and (b) on linear and log scales. Comparison with the data appears qualitatively quite good up to $\operatorname{Re}_{S} \approx 1$. As expected, however, comparison visually degrades at higher $\operatorname{Re}_{S} \gtrsim 1$ and $S^{*} \approx 200$. In an attempt to improve the comparison at higher $\operatorname{Re}_{S}$ and $S^{*}$, we added a nonlinear term to Eq. (11) to produce

$$
\ln \left(S h-S h_{0}\right)=a+b \ln (S *)+c \ln \left(\operatorname{Re}_{S}\right)+d \ln (S *) \ln \left(\operatorname{Re}_{S}\right) .
$$

This "correlation 2" has the following more complicated form in comparison with Eq. (10):

$$
S h=S h_{0}+e^{a}\left(S^{*}\right)^{b} \operatorname{Re}_{S}^{c+d \ln S^{*}} .
$$

Again using multivariable linear least squares regression on Eq. (12) we find the coefficients $a, b, c$ and d listed under "correlation 2" in Table 1 with the resulting correlation plotted in Figure 9 (c) and (d) on linear 
(a)

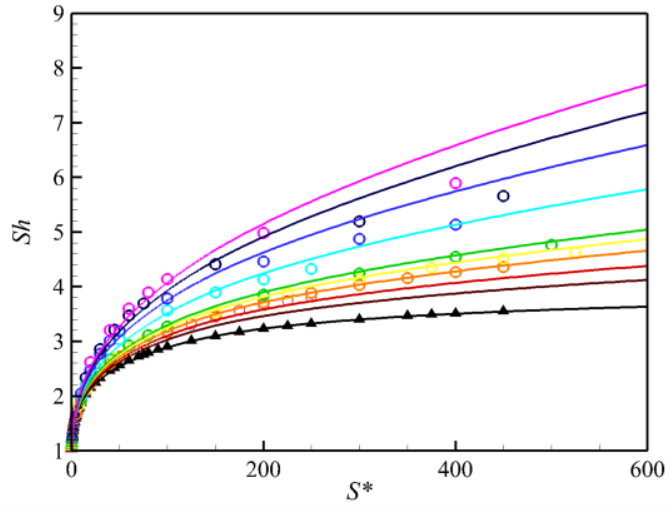

(c)

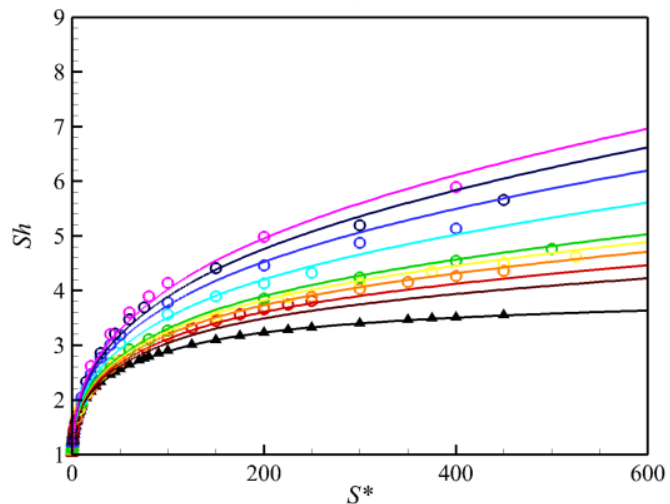

(b)

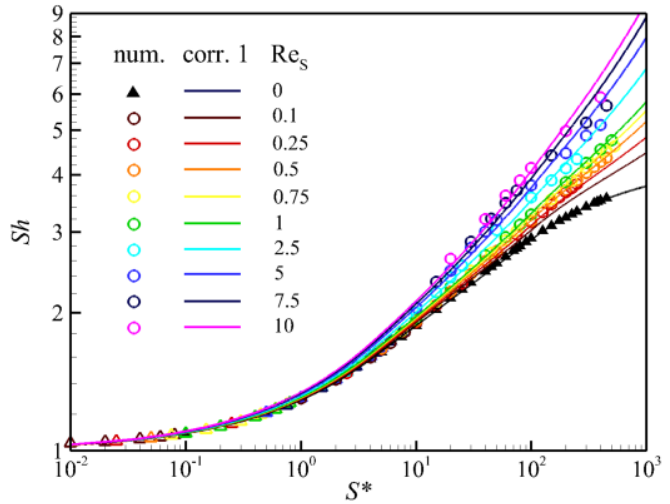

(d)

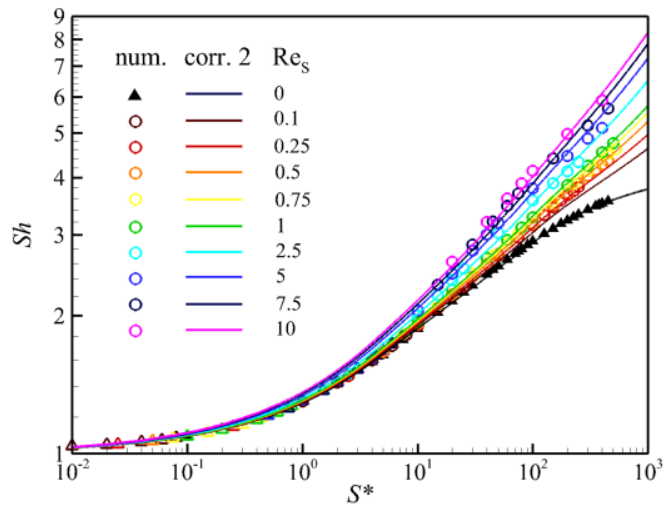

Figure 9. Comparison of computational data for $S h$ vs. $S^{*}$ at fixed $\operatorname{Re}_{S}>0$ with correlations 1 $(a, b)$ and $2(c, d)$ on linear-linear $(a, c)$ and $\log -\log (b, d)$ scales.

and $\log$ scales. Visually, the improvement is evident at higher $S^{*}$ and $\operatorname{Re}_{S}$ in comparison with correlation 1 - with the tradeoff of a significantly more complicated mathematical form for the correlation.

Inserting the coefficients in Table 1 into Eqs. (11) and (12) produces the final forms of the two nonzero $\operatorname{Re}_{S}$ correlations for Sherwood number as a function of Peclet and Reynolds numbers:

Correlation 1:

$$
S h=S h_{0}+0.01767\left(S^{*}\right)^{0.684} \operatorname{Re}_{S}^{0.460},
$$

Correlation 2:

$$
S h=S h_{0}+0.01874\left(S^{*}\right)^{0.674} \operatorname{Re}_{S}^{0.583-0.032 \ln S^{*}},
$$

where $S h_{0}=S h_{0}\left(S^{*}\right)$ is given by Eq. (9). The hydrodynamic enhancement to the Sherwood number from local shear-rate, $\Delta_{\text {shear }}$ in Eq. (1), is given by $\Delta_{\text {shear }}=S h-1$ with $S h$ given by Eqs. (14) or (15) with Eqs. (13) for the zero Reynolds number Sherwood number, $S h_{0}$.

We should point out that in the least squares fitting procedure it was necessary to alleviate a concern with data selection in the accuracy of the linear regression procedure. As discussed in context with Figure 5 , the influence of Reynolds number on the Sherwood number is negligible when $\operatorname{Re}_{S} \leqslant 0.1$ and $S^{*}<100-200$. Assume an error $\delta_{\text {err }}$ in the estimation of $\left(S h-S h_{0}\right)$. Then truncated Taylor series produces $\ln \left(S h-S h_{0}+\delta_{\text {err }}\right)=\ln \left(S h-S h_{0}\right)+\delta_{\text {err }} /\left(S h-S h_{0}\right)+\ldots$, which implies that $\ln \left(S h-S h_{0}\right)$ is 
amplified by the error divided by a large number, $1 /\left(S h-S h_{0}\right)$, in the Stokes flow limit. Given that there is always some error in $S h-S h_{0}$, we eliminated the data points in the regimes $\operatorname{Re}_{S} \lesssim 0.1$ and $S^{*} \leqslant 5$ from the regression procedure to improve accuracy.

\section{Analysis}

In Figure 10 we illustrate the quality of the fit to the computational data as a function of $S^{*}$ for fixed $\operatorname{Re}_{S}$ (Figure 10a) and as a function of $\operatorname{Re}_{S}$ for fixed $S^{*}$ (Figure 10b). Although the second correlation is superior at higher $S^{*}$ and $\operatorname{Re}_{S}$ over the ranges of $S^{*}$ and $\operatorname{Re}_{S}$ considered in this study, both correlations appear to reasonable predictions (keeping in mind the log axis), capturing the nonlinear features of the dependencies of Sherwood number on nondimensional shear rate, $S^{*}$ (Peclet number) and on shear Reynolds number, $\operatorname{Re}_{S}$. For example, the sensitivity in $S h$ to variations in $\operatorname{Re}_{S}$ increases with the increasing $S$, from negligible sensitivity at $\operatorname{Re}_{S}$ roughly below 1 and $S^{*}$ below roughly 10, to strong sensitivity at $S^{*}$ roughly over 100 .

It is apparent subjectively from Figures 9 and 10 that there is a trend in the correlations to depart from the data at the highest Peclet and Reynolds numbers. To quantify the extent to which the correlations we developed match the data, we plot in Figure 11 the relative difference between the data and correlation at each data point, for correlation 1 vs. correlation 2 . Several interesting observations can be made from this plot. Firstly, note that the relative difference is less than $2 \%$ at all $S^{*}$ when $\operatorname{Re}_{S}$ is $\approx 1$ or less and that there is very little difference between correlation 1 vs. correlation 2 . This is the typical scenario, at least for drug release from typical sized drug particles, as illustrated in Figure 5 for dissolution of typical ibuprofen particles in water, where shear Reynolds number never exceeded 0.01 in vivo and 0.3 in vitro. Thus, for many practical applications the simpler of the two correlations, Eq. (14), is more than adequate.

Secondly, we note that the curves at the Reynolds numbers of 2.5, 5, 7.5 and 10 produce a single group, separated from the lower Reynolds numbers, with a maximum relative difference between correlation and data of $6 \%$ with the more complicated correlation 2 (Eq. 15) and $12 \%$ with the simpler correlation (Eq. 14). However the $6 \%$ vs. $12 \%$ comparison is only a relevant comparison at the highest $S^{*}$ of 500 . At $S^{*} \lesssim 200$ the maximum difference between correlation and data is $4 \%$ for both correlations. Furthermore, we note that an unnatural oscillation in the curve $\delta_{\text {diff }}\left(S^{*} ; \operatorname{Re}_{S}\right)$ for $\operatorname{Re}_{S} \geq 2.5$ with peaks at $S^{*} \approx 50$ and 500 . We believe this nonphysical characteristic is indicative of numerical stability concerns at the highest simulated

(a)

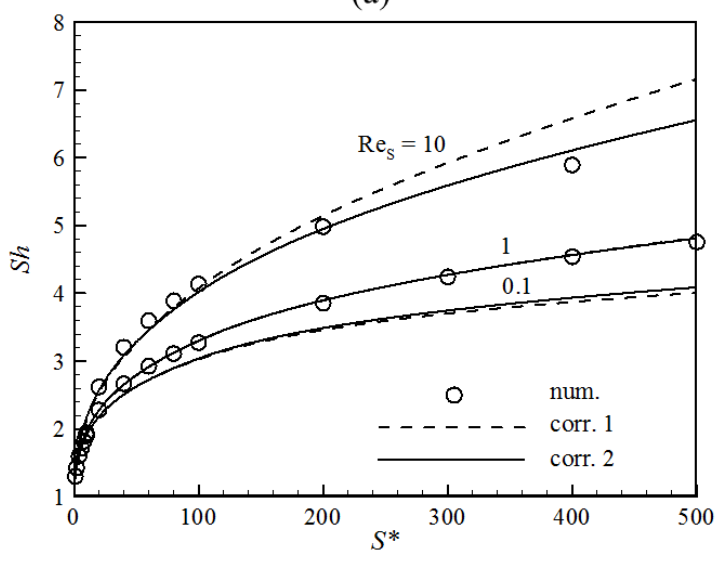

(b)

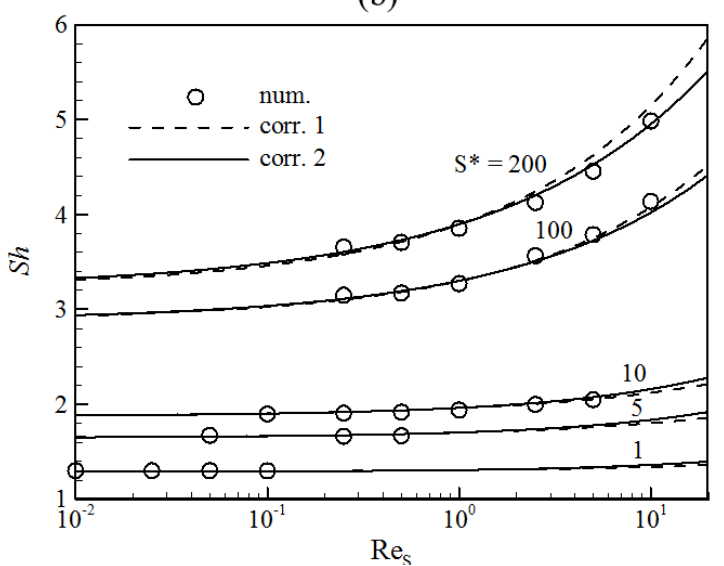

Figure 10. Detailed comparison of the two finite Reynolds number correlations with the computational data, (a) as a function of $S^{*}$ at fixed $\operatorname{Re}_{S}$, and (b) as a function of $\operatorname{Re}_{S}$ for fixed $S^{*}$. 
values of $S^{*}$ and $\operatorname{Re}_{S}$. For these simulations, grid resolution was increased by a factor of two in all directions in space and time, increasing the computational domain by a factor of two in all directions and an increase in computational load by a factor of over 16 for the highest $\left(S^{*}, \operatorname{Re}_{S}\right)$ simulations.

(a)

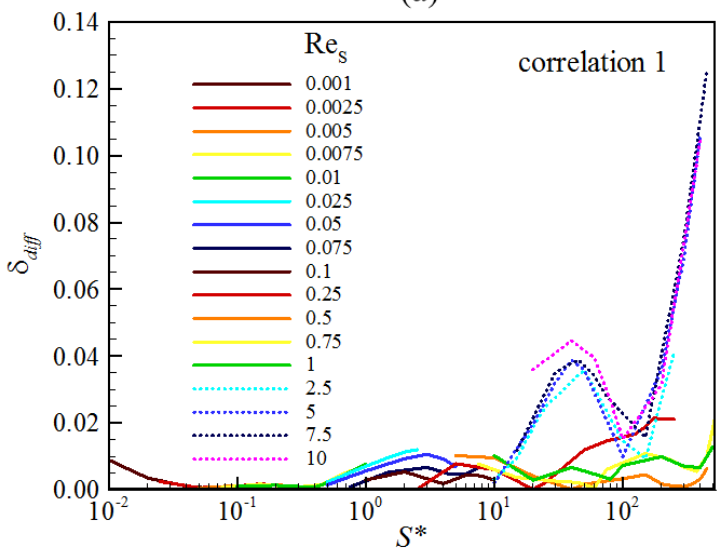

(b)

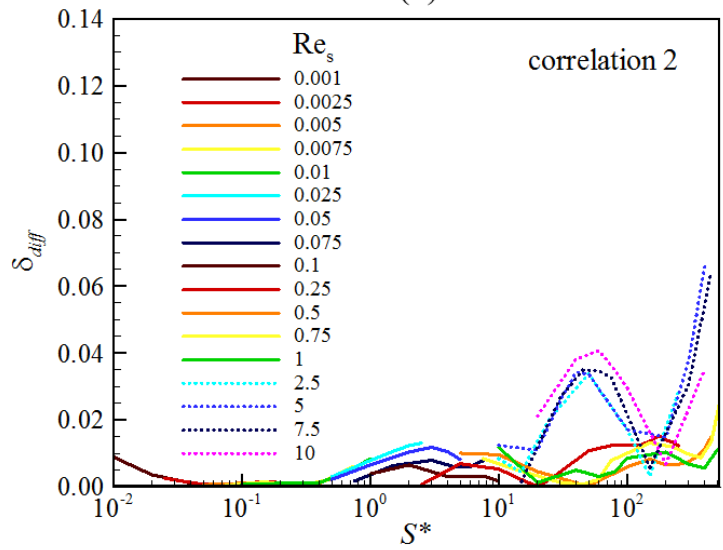

Figure 11. Relative difference $\delta_{\text {diff }}$ in Sh between data points and the empirical curve fits (correlations) at the same $S^{*}$ and $\operatorname{Re}_{S}$

\section{Sensitivity and Application}

In Appendix $C$ we quantify the sensitivities in the response of Sherwood number to changes in Peclet and Reynolds number. As has been discussed above in context with Figures 5 and 10, sensitivity in the response of Sherwood number to changes in Peclet and Reynolds number depends on the $S^{*}$ and $\operatorname{Re}_{S}$ regime, with greatest sensitivity to changes in $S^{*}$ and $\operatorname{Re}_{S}$ tending to occur in the lower and higher $S^{*}$ ranges, respectively.

In Appendix $C$ we conclude that the highest sensitivity to changes in $S^{*}$ occur roughly in the range $S^{*} \sim 0$ to 20 at all $\operatorname{Re}_{S}$, with an apparent singularity at $S^{*}=0$. The range in $S^{*}$ of high sensitivity increases gradually with increasing $\operatorname{Re}_{S}$. This is a significant result because the release of molecules from the surfaces of drug particles tends to occur at lower $S^{*}$ with $\operatorname{Re}_{S}$ approaching zero. The high sensitivity to changes in $S^{*}$ degrades rapidly with increasing $S^{*}$ to small values at $S^{*}$ exceeding roughly 200-300 at Reynolds numbers below $\sim 1$. However at these high $S^{*}$ values, the sensitivity to $S^{*}$ increases with increasing $\operatorname{Re}_{S} \gtrsim 1$, especially at $S^{*}$ exceeding $\sim 400-500$. The highest sensitivity of $S h$ to changes in $\operatorname{Re}_{S}$

occurs at very low $\operatorname{Re}_{S}$ (relative to 1) with an apparent singularity at $\operatorname{Re}_{S}=0$. The range in $\operatorname{Re}_{S}$ where $S h$ is highly sensitive to changes in $\operatorname{Re}_{S}$ increases with increasing $S^{*}$, particularly at small $\operatorname{Re}_{S}$ and $S^{*}>$ roughly 100 . Conversely, sensitivity to changes in $\operatorname{Re}_{S}$ is very low at $S^{*}$ below roughly 10 . Sensitivity drops rapidly with increasing $\operatorname{Re}_{S}$, especially at lower $S^{*}$. At $S^{*}<\sim 10$, there is negligible sensitivity to changes in $\operatorname{Re}_{S}$.

From an applications perspective, one might ask over what range of parameters the Stokes-flow correlations of Eq. (9) could be applied with good accuracy. In Appendix $C$ we conclude that the Stokes flow model is reasonable (within 10\%) over the entire $S^{*} \sim 0-500$ range when $\operatorname{Re}_{S}<0.01$, but is reasonable for $S^{*} \lesssim 100$ when $\operatorname{Re}_{S} \lesssim 0.5$. 


\section{Discussion: Relative Importance of Convective vs. Shear-rate Enhancements}

An issue of major interest within the pharmaceutical scientists mathematical modeling community is the prediction of mass transfer from thousands of drug particles in vivo, a key issue being hydrodynamic enhancement of dissolution rate compared to pure diffusion in a stagnant fluid. Related to this is the potential importance of dissolution in vivo vs. within the in vitro test devices commonly used to quantify bioequivalence. Of specific concern in the in vivo vs. in vitro comparison is difference in hydrodynamic influences on dissolution. Because drug particles typically enter the flow environment with diameters < $\sim 100 \mu \mathrm{m}$, and with the current trend towards "micronization," prediction methods for pharmaceutical applications generally focus on dissolution of "small particles" and the dissolution limit $R \rightarrow 0$.

Most mathematical models for drug dissolution are based on pure diffusion, sometimes with ad-hoc corrections based on an imprecisely-defined diffusion layer concept ${ }^{4}$. Wang et al. ${ }^{5}$ proposed a modeling strategy based on the normalized surface flux $S h$ (Eq. 1) with the extension given by Eq. (2) to include hydrodynamic effects. Historically the hydrodynamic influence discussed in the literature is "convection" or "slip," the enhancement of transport of mass or heat from a surface from relative velocity between the fluid and surface across which mass/heat flux takes place ${ }^{19}$. For application to drug dissolution, relative velocity between fluid and particle (slip velocity magnitude, $\Delta U$ ) is primarily a result of density difference between particle and surrounding fluid (inertia) and the finite size of the particle. As mentioned in the Introduction, the standard empirical correlation for convective enhancement in Sherwood number for small spherical particles was developed by Ranz and Marshall ${ }^{1}$, Eq. (3) in the Introduction, which shows that $\Delta_{\text {conv }} \sim P e_{\Delta u}^{1 / 3} \operatorname{Re}_{\Delta u}^{0.17} \sim[(\Delta U) R]^{1 / 2}$. Thus, to evaluate $\Delta_{\text {conv }}$, a model for slip velocity magnitude, $\Delta U \equiv|\Delta \mathbf{u}|$, in particular its scaling on $R$, is required.

Although drag force dominates particle weight on small drug particles, the slip velocity of drug particles is commonly incorrectly modeled as the settling velocity of the same particle in a stagnant fluid at steady state under the influence of gravity, a mechanism unrelated to the generation of slip velocity on drug particles in practice. In practice, the flows into which are embedded drug particles are driven in vivo by contractions of the intestinal wall (motility) and in the most common in vitro devices by the rotational motion of an impeller typically rotating 50-100 RPM. ${ }^{17}$ In Appendix A we develop a slip model for small spherical particles moving within a fluid flow to show that $\Delta U \sim R^{2}$ in the small particle limit, implying that $\Delta_{\text {conv }} \sim[(\Delta U) R]^{1 / 2} \sim R^{3 / 2}$.

The current study has led to the development of the correlations in Eqs. (9) and (14) or (15) for hydrodynamic enhancement associated with shear rate at the location of a dissolving particle. In the limit $R \rightarrow 0, \Delta_{\text {shear }} \sim S h_{0}-1 \sim \sqrt{S R^{2} / D_{m}} \sim R$, so that $\Delta_{\text {shear }} / \Delta_{\text {conv }} \sim 1 / \sqrt{R} \rightarrow \infty$ as $R \rightarrow 0$. Thus shear-rate enhancement dominates convective enhancement in the small-particle limit (e.g., as particles dissolve).

Appendix A contains a detailed analysis of the importance of convective and shear-rate enhancement of surface mass flux for particles over a wide range of radii. We show in Figure A5 the impact of both convective and shear-rate enhancement separately and relative to one another as a function of particle radius within a lattice-Boltzmann simulation of modeled intestinal flow parameterized to the human intestinal function. We conclude from this analysis that convective enhancement of dissolution rate is at most only a couple percent even for larger particles (diameters $d>200 \mu \mathrm{m}$ ), while enhancement by shear-rate is a leading order effect (10-50\% for $d \sim 40-200 \mu \mathrm{m})$ and fully dominates convective enhancement at all particle diameters up to a few hundred $\mu \mathrm{m}$, but especially as particles dissolve. Recent, as yet unpublished, computational predictions of drug release from thousands of ibuprofen drug particles within intestinal 
peristalsis $^{20}$ suggest that hydrodynamic enhancement from convection is negligible in comparison to hydrodynamic enhancement from shear-rate.

A fundamental distinction between hydrodynamic enhancement from shear-rate vs. convection is that, whereas convective enhancement disappears in Stokes flow (Eq. 3), enhancement from shear-rate can be strong at zero shear Reynolds number. Indeed, Figure 5 shows that, whereas shear-rate enhancement is insensitive to $\operatorname{Re}_{S}$ at small $S^{*}$, there exists a strong sensitivity to small increases in $S^{*}$ relative to dissolution in a stagnant fluid (i.e., pure diffusion). The essential mechanism underlying both the great sensitivity to shear-rate at low $S^{*}$ and the dominance of shear-rate enhancement over convective enhancement for small particles, is the generation of a highly three-dimensional flowfield adjacent to the particle surface that is induced by particle spin, as shown in Figures 2 and 3, together with the transfer of the released molecules from the particle by shear (Figure 4). Particle spin is itself is a response to the vortical content of shear flow, content unrelated to the slip mechanism. Figures 6 and 13 (a) show that even at low normalized shear rate $S^{*}$ local 3D spin-induced flow can generate major enhancements to surface mass (and heat) flux.

We conclude that "shear-rate enhancement" to mass and heat transfer from particles embedded within complex fluid flows, in comparison to mass/heat release within a stagnant fluid, may be important in a wide variety of scenarios - not only within the pharmaceutical sciences, but more broadly within a wide range of engineering technologies that involve heat or mass transport from large numbers of particles within a fluid flow. It would be of interest to apply the correlations developed here, together with the Ranz/Marshall correlations and accurate appropriate models for slip velocity (Appendix A), to evaluate relative contributions to these and other mechanisms for heat and mass transfer from spherical and non-spherical particles in a wide range of practical applications over a wide range of particle Peclet and Reynolds numbers.

\section{Acknowledgements}

We are grateful to the U.S. Food and Drug Administration (FDA) for financial support under contract no. FDA_RFQ1116088. We thank our colleagues, Greg and Gordon Amidon (University of Michigan), Bertil Abrahamsson (AstraZeneca Sweden), and Deanna Mudie (currently Lonza Pharma \& Biotech, formerly University of Michigan) for many useful discussions surrounding the research and developments described here.

\section{Literature Cited}

1. Ranz WE, Marshall WR, Evaporation from drops. Chem. Eng. Prog. 1952; 48: 141-146.

2. Lindfors L, Jonsson M, Weibull E, Brasseur JG, Abrahamsson B, Hydrodynamic effects on drug dissolution and deaggregation in the small intestine - a study with felodipine as a model drug. J. Pharm. Sci. 2015; 104: 2969-2976.

3. Amidon, G, Lennernäs, H., Shah, VP, Crison, JR, A theoretical basis for biopharmaceutic drug classification: the correlation on in vitro drug product dissolution and in vivo bioavailability. Pharm. Res. 1995, 12: 413-420.

4. Wang Y, Abrahamsson B, Lindfors L, Brasseur, JG, Comparison and analysis of theoretical models for diffusion-controlled dissolution. Mol. Pharm. 2012; 9: 1052-1066. 
5. Wang Y, Abrahamsson B, Lindfors L, Brasseur JG, Analysis of diffusion-controlled dissolution from polydisperse collections of drug particles with an assessed mathematical model. J. Pharm. Sci. 2015; 104: 2998-3017.

6. Han, X, Ghoroi, G, Chen, Y, Davé, Simultaneous micronization and surface modification for improvement of flow and dissolution of drug particles, Intern. J. Pharmaceutics 2011, 185-195.

7. Frankel NA, Acrivos A. Heat and mass transfer from small spheres and cylinders freely suspended in shear flow. Phys. Fluids. 1968; 11: 1913-1918.

8. Batchelor GK. Mass transfer from a particle suspended in fluid with a steady linear ambient velocity distribution. J. Fluid Mech. 1979; 95: 369-400.

9. Acrivos A. Heat transfer at high Peclet number from a small sphere freely rotating in a simple shear field. J. Fluid Mech. 1971; 46: 233-240.

10. Yang C, Zhang J, Koch DL, Yin X. Mass/heat transfer from a neutrally buoyant sphere in simple shear flow at finite Reynolds and Peclet numbers, AIChE J. 2011;.57(6): 1419-1433.

11. Polyanin AD, Dil'man VV. New methods of the mass and heat transfer theory II. The methods of asymptotic interpolation and extrapolation. Int. J. Heat Mass Transfer. 1985; 28(1): 45-58.

12. Subramanian G, Koch DL. Centrifugal forces alter streamline topology and greatly enhance the rate of heat and mass transfer from neutrally buoyant particles to a shear flow. Phys. Rev. Lett. 2006a; 96: 134503.

13. Subramanian G, Koch DL. Inertial effects on the transfer of heat or mass from neutrally buoyant spheres in a steady linear velocity field. Phys. Fluids. 2006b; 18: 073302.

14. Longest PW, Kleinstreuer C, Interacting effect of uniform flow, plane shear, and near-wall proximity on the heat and mass transfer of respiratory aerosols. Int. J. Heat Mass Trans. 2004; 47: 4745-4759.

15. Wang Y, Brasseur JG, Banco GG, Webb AG, Ailiani AC, Neuberger T. Development of a latticeBoltzmann method for multiscale transport and absorption with application to intestinal function. Computational Modeling in Biomechanics, Ch. 3. Springer, 2010.

16. Leal LG, Advanced Transport Phenomena - Fluid Mechanics and Convective Transport Processes. Cambridge University Press, 2007.

17. Kukura, J., Baxter, J.L., Muzzio, F.J. Shear distribution and variability in the USP Apparatus 2 under turbulent conditions. Intern. J. Pharmaceutics. 2004, 279: 9-17.

18. Banco, Gino G. 2010 Multi-Scale Fluid Mechanics of Nutrient Absorption in the Small Intestine with $2 D$ and $3 D$ Lattice-Boltzmann Models. Ph.D. Thesis, Department of Mechanical Engineering, Pennsylvania State University, University Park, PA, December 2010.

19. Sugano, K. Theoretical comparison of hydrodynamic diffusion layer models used for dissolution simulation in drug discovery and development. Intern. J. Pharmaceutics 2008, 363: 73-77.

20. Behafarid, F., Brasseur, JG. 2017 Hydrodynamic impacts on dissolution, transport and absorption from thousands of drug particles moving within the intestines. (abstract) Bull. Amer. Phys. Soc. 62 (14), November 2017. http://meetings.aps.org/link/BAPS.2017.DFD.D37.6. 


\section{APPENDIX A: \\ Convective vs. Shear-rate Enhancement with Application to in vivo Drug Dissolution, and a Slip Velocity Model}

As discussed in the Introduction, a primary application of the current study is the prediction of drug dissolution and differences in drug dissolution in the human intestines vs. in in vitro dissolution test devices commonly used in the pharmaceutical industry. The result from this study that shear-rate enhancement greatly dominates convective enhancement is of great importance to pharmaceutical modeling of drug dissolution. In this Appendix we provide the analysis underlying this important result. However to compare convective with shear enhancement requires a model for slip velocity that appears in the slip Peclet and Reynolds numbers, $P e_{\Delta U} \equiv(\Delta U) R / D_{m}$ and $R e_{\Delta U} \equiv(\Delta U) R / v$ upon which the slip enhancement to Sherwood number depends. We develop such a model in the first section of this Appendix in context with our application to drug dissolution in the intestines.

Our focus is on the ranges of $P e_{\Delta U}$ and $R e_{\Delta U}$ encountered by individual drug particles as they move within the intestinal fluid, approximately Newtonian incompressible fluid motions driven by the timevarying deformations of the intestinal wall. The intestinal wall contain neutrally controlled muscle fibers to produce contractile patterns that force patterned fluid motions within the intestinal lumen. The most common contractile patterns are peristaltic and segmental. To identify the ranges of $\Delta U$ that typical drug particles experience in vivo, we briefly describe in the second section of this Appendix lattice-Boltzmann CFD of peristalsis and segmental contraction in the human intestines from which we estimated flowfields and ranges of $\Delta U$ encountered by typical ranges of drug particle sizes in vivo.

Based on the first two sections in this Appendix, in the third section we present an analysis of the relative magnitudes of slip velocity enhancement of drug dissolution (convection) vs. shear-rate enhancement. This analysis is central to the final section of the manuscript entitled "Discussion: Relative Importance of Convective vs. Shear-rate Enhancements" where we argue that shear-rate enhancement strongly dominates convective enhancement in the small-particle limit for drug-particle dissolution in vivo. Here we explain this result in more detail for a range of particle sizes relevant to intestinal dissolution from drug particles.

Please note that references specific to each Appendix are placed at the end of the appendix.

\section{Model for Slip Velocity}

In context with the flow environment associated with intestinal motility, described in more detail in the next section, we develop a model for $\Delta U \equiv|\Delta \mathbf{u}|$ for use within the Ranz/Marshall ${ }^{2}$ correlation for convective enhancement to the Sherwood number given by Eq. (3) in the body of the manuscript as a function of slip Peclet and Reynolds numbers. Our model is based on the "point" particle model of Maxey and Riley ${ }^{1}$ which assumes particles small relative to the characteristic eddying scales of fluid motion (these scale on $R_{\max }$ ). In practice, our interest in dissolving drug particles focuses our attention on maximum particle radii 100-300 $\mu \mathrm{m}$ within intestinal lumen having characteristic radial dimensions $\sim 1-2 \mathrm{~cm}$ after a meal (the "fed state") and with characteristic collapse speeds $c \sim 1-2 \mathrm{~cm} / \mathrm{s}$ in the intestines (see next section, Figure A1).

From an extensive history of development in the literature, Maxey and Riley ${ }^{1}$ developed the following Newton's-law-based model for the acceleration of point particles: 


$$
\begin{aligned}
\frac{4}{3} \pi R^{3} \rho_{P} \frac{d \mathbf{V}}{d t}= & \frac{4}{3} \pi R^{3}\left(\rho_{P}-\rho_{F}\right) \mathbf{g}+\frac{4}{3} \pi R^{3} \rho_{F} \frac{D \mathbf{u}}{D t}+\frac{2}{3} \pi R^{3} \rho_{F} \frac{d}{d t}\left[\mathbf{u}-\mathbf{V}+\frac{1}{10} R^{2} \nabla^{2} \mathbf{u}\right] \\
& +6 \pi R v \rho_{F}\left[\mathbf{u}-\mathbf{V}+\frac{1}{6} R^{2} \nabla^{2} \mathbf{u}\right]+6 R^{2} \rho_{F} \sqrt{\pi v} \int_{0}^{t} \frac{1}{\sqrt{t-\tau}} \frac{d}{d \tau}\left[\mathbf{u}-\mathbf{V}+\frac{1}{6} R^{2} \nabla^{2} \mathbf{u}\right] d \tau
\end{aligned}
$$

In this equation $\mathbf{V}$ and $\mathbf{u}$ are particle and fluid velocity (at the same point), $R$ is particle radius, $\rho_{F}$ and $\rho_{P}$ are fluid and particle density, $v$ is fluid kinematic viscosity, $\mathbf{g}$ is the gravitational acceleration and $t$ is time. The operators $d / d t$ and $D / D t$ denote time derivatives following the solid and fluid particles respectively (at the same point). The terms on the right-hand side of Eq. (A1) represent contributions from buoyancy force, fluid acceleration, added mass, Stokes drag, and "Basset history" which models memory effects (see Maxey and Riley ${ }^{1}$ ).

We follow the analysis of Lasheras \& Tio $^{5}$ based on the following nondimensional parameters

$$
\varepsilon \equiv \frac{\rho_{F}}{\rho_{P}+0.5 \rho_{F}}=\frac{1}{\rho_{P} / \rho_{F}+0.5}, \quad S t \equiv \frac{2 R^{2} U}{9 \varepsilon v L}, \quad \mathbf{G} \equiv \frac{L \mathbf{g}}{U^{2}},
$$

where $L \sim R_{\max } \sim 1-2 \mathrm{~cm}$ and $U \sim c \sim 1-2 \mathrm{~cm} / \mathrm{s}$ are the characteristic fluid flow length and velocity scales ( $R$ is particle radius as before). $S t$ is the particle Stokes number. $\varepsilon$ parameterizes particle-to-fluid density ratio. Drug particles typically have particle-to-fluid density ratios in the range $\rho_{P} / \rho_{F} \sim 0.5-2$, implying $\varepsilon \sim 1-0.4$, with neutrally buoyant particles given by $\varepsilon=2 / 3$. One may insert these characteristic ranges of parameters into the relation for Stokes number (Eq. A2) to find that for typical drug particles in vivo, St $\ll 1$.

Normalizing spatial coordinates, velocity, and time scales as

$$
\tilde{\mathbf{x}}=\frac{\mathbf{x}}{L}, \tilde{\mathbf{u}}=\frac{\mathbf{u}}{U}, \tilde{\mathbf{v}}=\frac{\mathbf{v}}{U}, \tilde{t}=\frac{t U}{L},
$$

Eq. (A1) becomes ${ }^{36}$

$$
\begin{aligned}
\frac{d \tilde{\mathbf{V}}}{d t} & =\frac{1}{S t}(\tilde{\mathbf{u}}-\tilde{\mathbf{V}})+\left(1-\frac{3}{2} \varepsilon\right) \mathbf{G}+\varepsilon\left(\frac{D \tilde{\mathbf{u}}}{D \tilde{t}}+\frac{1}{2} \frac{d \tilde{\mathbf{u}}}{d \tilde{t}}\right) \\
& +\frac{1}{S t^{1 / 2}} \frac{3}{\sqrt{2 \pi}} \varepsilon^{1 / 2} \int_{0}^{\tilde{t}} \frac{1}{\sqrt{\tilde{t}-\tilde{\tau}}}\left(\frac{d \tilde{\mathbf{u}}}{d \tilde{\tau}}-\frac{d \tilde{\mathbf{V}}}{d \tilde{\tau}}\right) d \tilde{\tau}
\end{aligned}
$$

Following Lasheras \& $\mathrm{Tio}^{5}$, we expand the particle velocity for $S t \ll 1$ as

$$
\tilde{\mathbf{V}}=\sum_{n=0}^{\infty} S t^{n / 2} \tilde{\mathbf{V}}^{(n)}(\tilde{\mathbf{x}}, \tilde{t})
$$

Substituting into Eq. (A5) and collecting terms of the same order in St we obtain the following results for $\mathbf{V}^{(n)}(\mathbf{x}, t)$ to $n=4$ :

$$
\begin{gathered}
\tilde{\mathbf{V}}^{(0)}=\tilde{\mathbf{u}}+\frac{\tilde{R}^{2}}{6} \tilde{\nabla}^{2} \tilde{\mathbf{u}} \\
\tilde{\mathbf{V}}^{(1)}=0
\end{gathered}
$$




$$
\begin{gathered}
\tilde{\mathbf{V}}^{(2)}=\left(\frac{3}{2} \varepsilon-1\right)\left(\frac{D \tilde{\mathbf{u}}}{D \tilde{t}}-\mathbf{G}\right)-\frac{\tilde{R}^{2}}{6}\left(1-\frac{3}{10} \varepsilon\right) \frac{D\left(\tilde{\nabla}^{2} \tilde{\mathbf{u}}\right)}{D \tilde{t}}-\frac{\tilde{R}^{2}}{6}\left(1-\frac{1}{2} \varepsilon\right) \tilde{\nabla}^{2} \tilde{\mathbf{u}} \cdot \tilde{\nabla} \tilde{\mathbf{u}} \\
\tilde{\mathbf{V}}^{(3)}=-\frac{3}{\sqrt{2 \pi}} \varepsilon^{1 / 2} \int_{0}^{\tilde{t}} \frac{\partial \tilde{\mathbf{V}}^{(2)} / \partial \tilde{\tau}^{+} \tilde{\mathbf{V}}^{(0)} \cdot \tilde{\nabla} \tilde{\mathbf{V}}^{(2)}}{\sqrt{\tilde{t}-\tilde{\tau}}} d \tilde{\tau}
\end{gathered}
$$

Equations (A6) - (A9) characterize the dominant impacts on particle velocity. Since the effect of the Basset history first appears in $\mathbf{V}^{(3)}$ at $O\left(S t^{3 / 2}\right)$, it is neglected in comparison to those of the other forces acting on the particle. Combining the other terms into (A5) yields the following expression for normalized slip velocity $\Delta \tilde{\mathbf{u}} \equiv \tilde{\mathbf{V}}-\tilde{\mathbf{u}}$ :

$$
\begin{aligned}
\Delta \tilde{\mathbf{u}}= & \frac{\tilde{R}^{2}}{6} \tilde{\nabla}^{2} \tilde{\mathbf{u}}+S t\left(1-\frac{3}{2} \varepsilon\right) \mathbf{G}-S t\left(1-\frac{3}{2} \varepsilon\right) \frac{D \tilde{\mathbf{u}}}{D \tilde{t}} \\
& -S t\left[\frac{\tilde{R}^{2}}{6}\left(1-\frac{3}{10} \varepsilon\right) \frac{D\left(\tilde{\nabla}^{2} \tilde{\mathbf{u}}\right)}{D \tilde{t}}+\frac{\tilde{R}^{2}}{6}\left(1-\frac{1}{2} \varepsilon\right) \tilde{\nabla} \tilde{\mathbf{u}} \cdot \tilde{\nabla} \tilde{\mathbf{u}}\right]+O\left(S t^{3 / 2}\right)
\end{aligned}
$$

Eq. (A10) produces an estimate for normalized slip velocity $\Delta \tilde{\mathbf{u}} \equiv \tilde{\mathbf{V}}-\tilde{\mathbf{u}}$ to order $S t^{3 / 2}$. The first term, the leading order term, characterizes particle drag, whereas the second term, buoyancy force, exists only when particle density differs from fluid density. The third term models response to fluid inertia while the final two terms, organized into a single bracketed term, reflects consequences of finite particle size. Using the lattice-Boltzmann based simulations described earlier for peristaltic transport relevant to intestinal motility (and additionally simulations of segmental contraction), we estimated each of the terms in Eq. (A10) for drug particles over a wide range of particle diameters and density ratios. We find that whereas the second finite-size term is fully negligible even extending outside intestinal flow ranges, the finite-size term that contains the material derivative of the velocity Laplacian can be significant within human intestinal motility for particles with diameter exceeding roughly $200 \mu \mathrm{m}$ and particle densities roughly twice the fluid density or higher. To make the model more precise, we retain this term.

Therefore, dropping only the second finite-size term in (A10) and unwrapping the normalized form of the equation, our model for slip velocity $\Delta \mathbf{u} \equiv \mathbf{V}-\mathbf{u}$ in dimensional form is:

$$
\Delta \mathbf{u} \approx \frac{R^{2}}{6} \nabla^{2} \mathbf{u}+\frac{2 R^{2}}{9 \varepsilon v}\left(1-\frac{3}{2} \varepsilon\right) \mathbf{g}-\frac{2 R^{2}}{9 \varepsilon v}\left(1-\frac{3}{2} \varepsilon\right) \frac{D \mathbf{u}}{D t}-\frac{R^{4}}{27 \varepsilon v}\left(1-\frac{3}{10} \varepsilon\right) \frac{D\left(\nabla^{2} \mathbf{u}\right)}{D t}
$$

We have used this model to access convective hydrodynamic enhancement to dissolution in comparison to the enhancements by shear-rate as the primary focus of the current manuscript. This analysis is carried out in context with characteristic intestinal flowfields developed with CFD as described in the next section.

\section{Characteristic Intestinal Flowfields}

We developed our model for $\Delta \mathbf{u}$ specific to drug dissolution in the human intestinal flow environment where the flow is driven in vivo by patterned contractions of the muscles within intestinal wall, referred to as "motility." Intestinal motility has two primary characteristics (in addition to quiescence): (1) peristalsis, or local axial mass movement of intestinal liquid by a traveling wall-contraction-wave pattern along the intestinal lumen, and (2) standing wave patterns that generate local mixing (without net transport) by 
spatially alternating local contractions of the intestinal wall within segments, what we refer to as "segmental contractions." The characteristic velocity and length scales of the patterns are similar in vivo as are the characteristic ranges of local velocity, strain-rate, vorticity and shear rate magnitudes. Although we have modeled both patterns and we reference the broader analysis for comparisons, to minimize space we summarize in this section CFD results for peristalsis.

We model peristalsis as illustrated in Figure A1 in a frame of reference moving with a sinusoidalshaped peristaltic contraction wave moving to the right with constant wave speed $c=2 \mathrm{~cm} / \mathrm{s}$, max radius $R_{\max }=1.5 \mathrm{~cm}$, and wavelength $\lambda=6 \mathrm{~cm}$, numbers consistent with characteristic motility in the human intestines ${ }^{3}$. Applying periodic boundary conditions in the axial direction $x$ we effectively calculate the fluid motions within an infinite number of repetitive sinusoidal "pockets" of intestinal liquid. The internal fluid velocity field drives flow radially towards and away from the tube surface and centerline velocity that scales on $c$. Whereas esophageal peristalsis is fully occluding, that is not the case in the intestines in the fed state; we chose $\delta / R_{\max }=0.11$ consistent with quantifications in the rat intestines ${ }^{35}$. Intestinal (Newtonian) fluid viscosity is 1010 times water and fluid density is that for water at $20 \mathrm{C}$. See $\mathrm{Banco}^{3}$ for discussion on these parameters from the gastro-intestinal literature and for additional modeling detail specific to intestinal motility. The lattice-Boltzmann method described in the section "Numerical Model" of the manuscript, and in more detail in Wang et al. ${ }^{4}$, was applied to solve the flow field in the stationary state from which the different terms in the particle acceleration equation presented in Maxey and Riley ${ }^{1}$ were compared to justify our reduced model for $\Delta \mathbf{u}$.

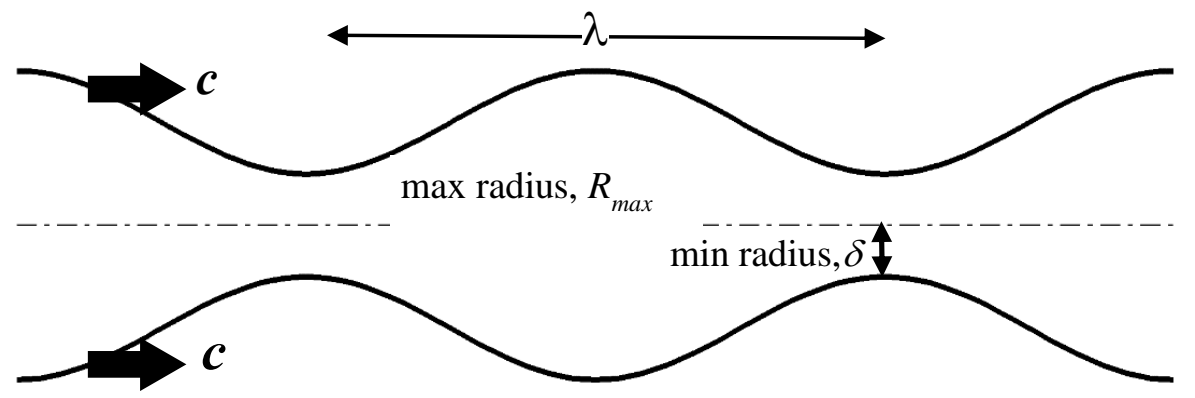

Figure A1. Physical models of the peristaltic contractile pattern used in our computational fluid dynamics (CFD) model to characterize the hydrodynamic flow fields experienced by drug particles in the small intestines. The modeled shape of the intestinal wall is axisymmetric (circular in cross section) and sinusoidal. The sinusoidal pattern propagates to the right with wave speed $c$ from strictly vertical motions of each material wall elements. All geometry and fluid motions are axisymmetric predicted with a 3D lattice-Boltzmann code. 
Figure A2 shows instantaneous streamlines associated with peristaltic contraction in the steady frame of reference moving with the contraction waves (a) and associated shear-rate distribution (b). Note that peristalsis is associated with a recirculation region in which particles are confined and move with the propagating wave. Whereas maximum shear-rate is of order 10-20 $\mathrm{s}^{-1}$ (Figure A2(b)), the most probable shear-rate is closer in the range 4-5 s-1. (Similar ranges for shear rate are found with segmental contraction.) Flow quantities required for the estimation of relative velocity and flow shear rate of solid particles within were acquired from the simulated flow fields developed for peristalsis and compared to corresponding simulations for segmental contraction. In the next section we analyze the relative contributions of convection vs. shear-rate enhancement to using the slip velocity model applied to the CFD flowfields described in the current section.

(a)

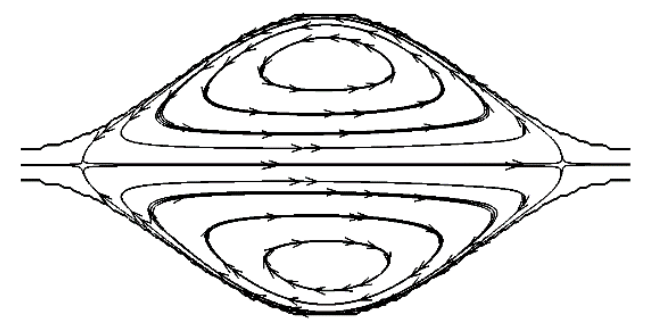

(b)

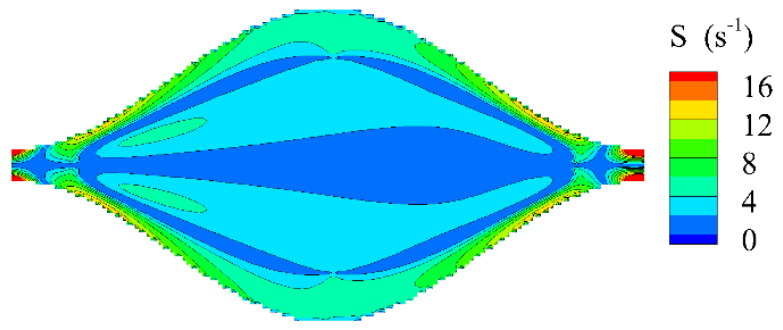

Figure A2. (a) shows instantaneous streamlines and (b) shows isocontours of instantaneous shear rate in a frame of reference moving with the peristaltic wave at wave-speed $c$, the steady frame of reference. $\delta / R_{\max }=0.11, c=2 \mathrm{~cm} / \mathrm{s}$.

\section{Analysis of Convective vs. Shear-rate Enhancement with Application to Drug Particle Dissolution}

From our lattice Boltzmann simulations of intestinal peristalsis (Figure A1), all terms in Eq. (A11) were calculated at all points in the computational domain over wide ranges of particle radii and density ratio. To estimate the overall enhancement to particle dissolution from slip velocity (convection), we averaged relevant quantities spatially over the computational domain and temporally over a single wave period. (Since $\Delta \mathbf{u}$ is frame-invariant, spatial averages in the steady frame of reference of Figure (A1) are equivalent to space-time averages in nonsteady frames.) For solid particle with radius $R$, the slip and shear-rate Reynolds numbers based on space-time averaged relative velocity $\langle\Delta \mathbf{u}\rangle$ and shear rate $\langle S\rangle$ were defined as

$$
\operatorname{Re}_{\Delta U} \equiv \frac{R|\langle\Delta \mathbf{u}\rangle|}{v}, \operatorname{Re}_{S} \equiv \frac{R^{2}\langle S\rangle}{v},
$$

where point-wise flow shear rate $S$ was calculated by first identifying the direction of the local flow streamline at each point and then calculating the magnitude of the transverse velocity gradient. That is, $S=\left|\partial u_{s} / \partial n\right|$ with $s$ and $n$ are the local streamwise and transverse directions, respectively.

The dependence of $\operatorname{Re}_{\Delta U}$ on particle radius $R_{P}$ and density ratio $\rho_{p} / \rho_{f}$ in our intestinal peristalsis model (Figure A1) is presented in Figure A3. Note that the isocontours are symmetric about $\rho_{p} / \rho_{f}=1$ and that slip Reynolds number increases with increasing $R$ at fixed $\rho_{p} / \rho_{f}$ and with $\left|\rho_{p} / \rho_{f}-1\right|$ at fixed 
$R$. Note also that the fact that $\operatorname{Re}_{\Delta U} \neq 0$ when particles are neutrally buoyant $\left(\rho_{p} / \rho_{f}=1\right)$ reflects finite particle size on slip velocity.

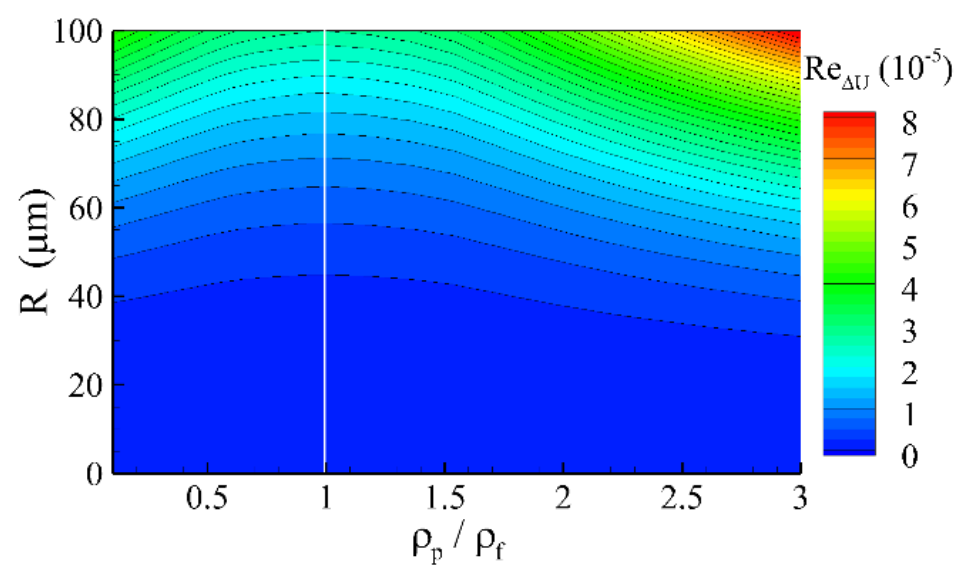

Figure A3. Dependence of $\operatorname{Re}_{\Delta U}$ on particle radius and density ratio with peristalsis with a liquid with the density of water at $20 \mathrm{C}$ and viscosity of $10 \mathrm{cps} . \delta / R_{\max }=0.11$ and $c=2 \mathrm{~cm} / \mathrm{s}$.

We define hydrodynamic enhancements to the Sherwood number (normalized flux) as $\Delta$. The Ranz/Marshall correlation for hydrodynamic slip enhancement of normalized surface flux (Sherwood number, $S h$ ) was given in the manuscript (Eq. 3): $\Delta_{\text {conv }}=0.424 P e_{\Delta u}^{1 / 3} \operatorname{Re}_{\Delta u}^{0.17}$, where Peclet number $\mathrm{Pe}_{\Delta U}=S c \mathrm{Re}_{\Delta U} \sim 1000 \mathrm{Re}_{\Delta U}$ with Sc being the Schmidt number (typically $\sim 10^{3}$ for dissolution of drug molecules in intestinal fluid). Thus, both slip Reynolds number and Peclet number directly impact enhancement. Figure (A3) indicates that particle slip Reynolds numbers are extremely small for particle diameters up to $200-300 \mu \mathrm{m}(R \sim 100-150 \mu \mathrm{m}): \max \operatorname{Re}_{\Delta U}$ is of order $10^{-4}$ for the largest and heaviest particles in the density range $\rho_{p} / \rho_{f} \sim 0.5-2$, and more typically $\sim 10^{-5}$ for dissolving particles less than $100 \mu \mathrm{m}$ in diameter. As importantly, these extremely low slip Reynolds numbers imply that maximum particle Peclet numbers are typically $~ 0.01-0.1$, with Peclet numbers of dissolving micronized drug particles much lower. Thus, the Ranz/Marshall correlation indicates maximum enhancement of drug particle dissolution in the intestines of order $\Delta_{\text {conv }} \sim 0.01-0.04$. We conclude that the influence of convection on hydrodynamic enhancement of drug dissolution in vivo is likely small or negligible.

Unlike the impact of convection on hydrodynamic enhancement of dissolution which disappears at zero slip Reynolds number, hydrodynamic enhancement from local flow shear-rate has a strong dependence on shear Peclet number $S^{*}$ at zero shear Reynolds number $\operatorname{Re}_{S}$ (Eq. (9) in the manuscript) that is quite sensitive to changes in $S^{*}$ at low $S^{*}$. Figure A4 shows that, although both $\operatorname{Re}_{S}$ and $S^{*} \sim 10^{3} \operatorname{Re}_{S}$ asymptotically approach zero in the limit $R \rightarrow 0, S^{*}$ is not small (relative to 1 ) for typical drug particle sizes in intestinal flow. Note that $S^{*}$ and $\operatorname{Re}_{S}$ are independent of $\rho_{p} / \rho_{f}$. 


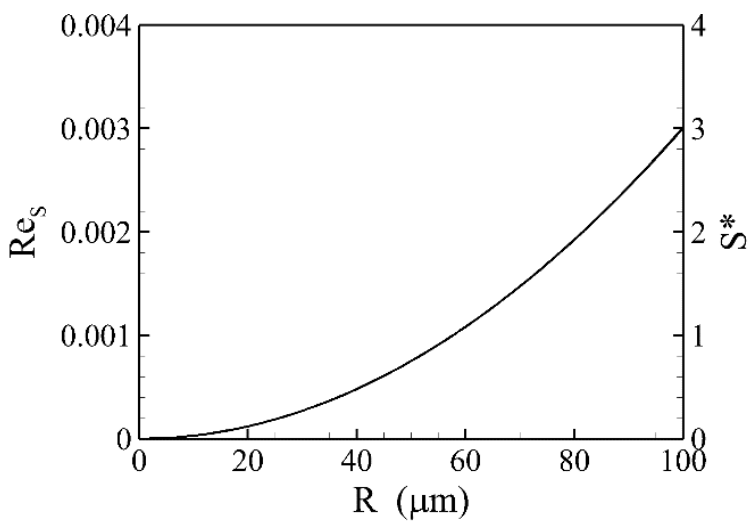

Figure A4. Dependence of $\operatorname{Re}_{S}$ and $\mathrm{S}^{*}$ on particle radius within liquid at the density of water at $20 \mathrm{C}$ and viscosity of $10 \mathrm{cps}$ for peristaltic contractions with $\delta / R_{\text {max }}=0.11, c=2 \mathrm{~cm} / \mathrm{s}, \mathrm{Sc}=1000$.

Using the correlations for $\operatorname{Sh}\left(S^{*}, \operatorname{Re}_{S}\right)$ in unbounded shear flow given by Eq. (15) in the manuscript, which use the zero Reynolds number correlation $S h_{0}\left(S^{*}\right)$ given by Eq. (9), we estimated averaged hydrodynamic enhancement to the Sherwood number from shear-rate, $\Delta_{c o n v},=S h-1$, using the Peclet and Reynolds numbers defined by Eq. (A12) for the simulations of intestinal peristalsis described earlier. These are compared with hydrodynamic enhancement from convection, $\Delta_{\text {conv }}$, in Figure A5. In Figure A5(a) $\Delta_{\text {conv }}$ and $\Delta_{\text {conv }}$ are plotted separately against particle radius $R$ to show that whereas convective enhancement to discussion is at most a couple percent for the larges particles and fully negligible for more typical drug particles, enhancement from shear-rate is a significant effect typically above $10 \%$ and as much as $50 \%$ for the largest drug particles. Whereas (a) shows that both effects disappear in the limit $R \rightarrow 0$, Figure A5(b) shows that hydrodynamic enhancement by shear rate fully dominates convective enhancement as drug particles dissolve. Furthermore, shear enhancement dominates convective enhancement to over an order of magnitude even in the large-particle limit. We repeated this analysis using computer simulations of

(a)

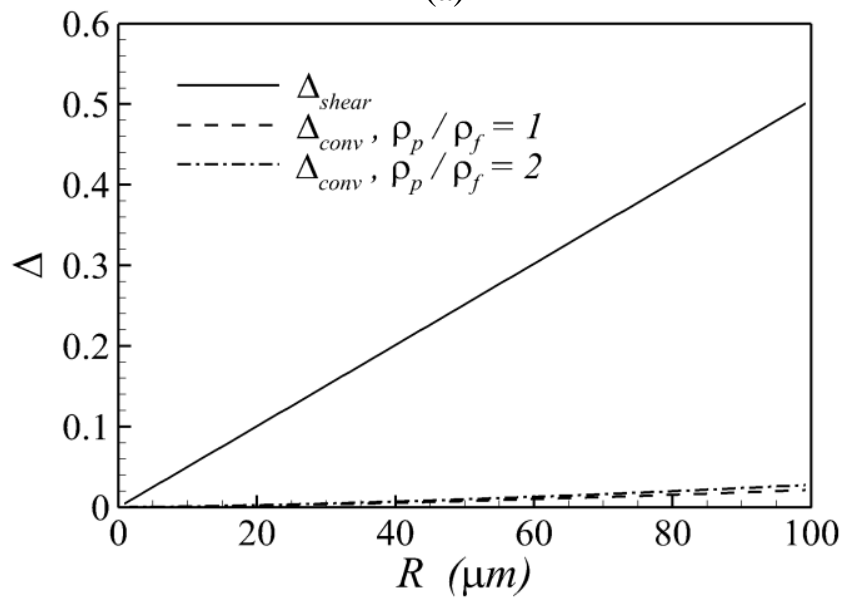

(b)

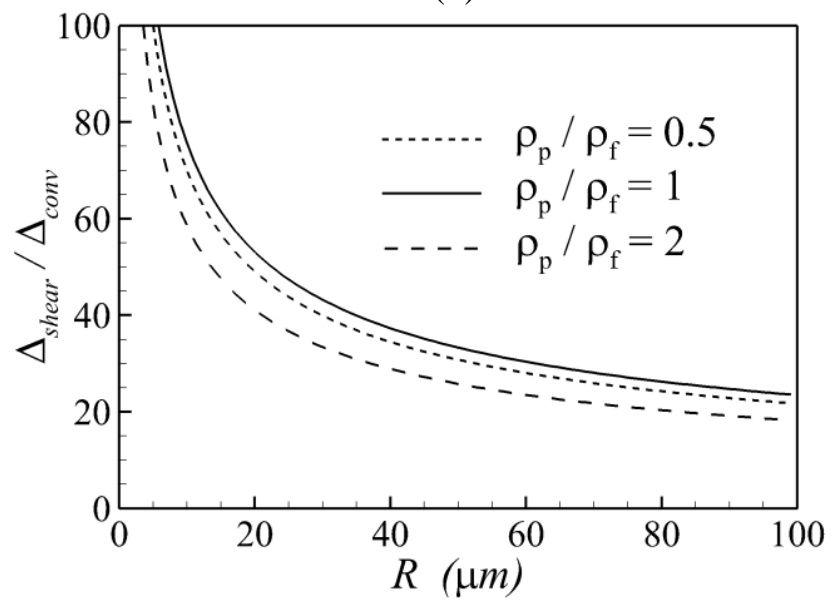

Figure A5. (a) Hydrodynamic augmentation to Sherwood number $(\Delta)$ from shear-rate $\left(\Delta_{\text {shear }}\right)$ and convection $\left(\Delta_{\text {conv }}\right)$; (b) Ratio of hydrodynamic augmentation from shear-rate relative to convection $\left(\Delta_{\text {shear }} / \Delta_{\text {conv }}\right)$ as a function of particle radius and particle-to-fluid density ratio. Peristaltic contractions, $\delta / R_{\text {max }}=0.11, c=2 \mathrm{~cm} / \mathrm{s}, \mathrm{Sc}=1000$. 
intestinal segmental contractions parameterized for the human intestines to draw the same conclusions as describe here for peristaltic intestinal motility.

We conclude that for application to drug dissolution in vivo hydrodynamic enhancement is important, convective enhancement is small or negligible, and shear enhancement fully dominates convective enhancement with the dominance increasing as the drug particle dissolves. Indeed, as pointed out in the "Discussion and Concluding Remarks" section of the manuscript, because $\Delta_{\text {conv }} \sim P e_{\Delta u}^{1 / 3} \operatorname{Re}_{\Delta u}^{0.17} \sim[(\Delta U) R]^{1 / 2}$ and, from Eq. (A11) $\Delta U \sim R^{2}, \Delta_{\text {conv }} \sim R^{3 / 2}$. In contrast, Eqs. (19) and (24) or (25), in the limit $R \rightarrow 0$, $\Delta_{\text {shear }} \sim R$ so that $\Delta_{\text {shear }} / \Delta_{\text {conv }} \sim 1 / \sqrt{R}$ in the limit $R \rightarrow 0$, consistent with Figure A5(b).

We conclude that for application to drug dissolution in vivo hydrodynamic enhancement is important, convective enhancement is small or negligible, and shear enhancement fully dominates convective enhancement with the dominance increasing as the drug particle dissolves. Indeed, as pointed out in the "Discussion and Concluding Remarks" section of the manuscript, because $\Delta_{\text {conv }} \sim P e_{\Delta u}^{1 / 3} \operatorname{Re}_{\Delta u}^{0.17} \sim[(\Delta U) R]^{1 / 2}$ and, from Eq. (A11) $\Delta U \sim R^{2}, \Delta_{\text {conv }} \sim R^{3 / 2}$. In contrast, Eqs. (19) and (24) or (25), in the limit $R \rightarrow 0$, $\Delta_{\text {shear }} \sim R$ so that $\Delta_{\text {shear }} / \Delta_{\text {conv }} \sim 1 / \sqrt{R}$ in the limit $R \rightarrow 0$, consistent with Figure A5(b).

\section{References for Appendix A}

1. Maxey, MR., Riley, JJ. Equation of motion for a small rigid sphere in a nonuniform flow. Phys. Fluids 1983, 26: 883-889.

2. Ranz WE, Marshall WR, Evaporation from drops. Chem. Eng. Prog. 1952; 48: 141-146.

3. Banco, Gino G. 2010, Multi-Scale Fluid Mechanics of Nutrient Absorption in the Small Intestine with 2D and 3D Lattice-Boltzmann Models. Ph.D. Thesis, Department of Mechanical Engineering, Pennsylvania State University, University Park, PA, December 2010.

4. Wang Y, Brasseur JG, Banco GG, Webb AG, Ailiani AC, Neuberger T. Development of a latticeBoltzmann method for multiscale transport and absorption with application to intestinal function. Computational Modeling in Biomechanics. Springer, 2010.

5. Lasheras JC, Tio KK. Dynamics of a small spherical particle in steady two-dimensional vortex flows. Appl. Mech. Rev. 1994; 47: S61-S69. 


\section{APPENDIX B: \\ Details of the Numerical Method and the Stokes Flow Limit}

\section{The Basic Lattice-Boltzmann Numerical Method for Momentum}

Description of the lattice-Boltzmann numerical method relevant to the current application is given in Wang, et al. ${ }^{1}$, which also describes validation experiments for the multigrid strategy and moving boundary conditions in 2D. Excellent reviews of several of the issues discussed in this section are given by Chen and Doolen $^{2}$ and Aidun and Clausen ${ }^{3}$.

As described in more detail in Wang, et al., we developed a 3D numerical method based on a multigrid strategy within the lattice-Boltzmann (LB) framework. The LB method for evolution of the momentum field can be viewed as an explicit finite difference representation of the continuous Boltzmann equation (Shan, et al. ${ }^{4}$ ) with the dependent variable being a particle distribution function $f(\mathbf{x}, \boldsymbol{\xi}, t)$ that quantifies the probability of an ensemble of fluid molecules at position $\mathbf{x}$ with velocity $\xi$ at time $t$. Continuum-level velocity $\mathbf{u}(\mathbf{x}, t)$ and density $\rho(\mathbf{x}, t)$ are obtained from moments of $f(\mathbf{x}, \xi, t)$ over velocity-space $\xi$. Distribution functions and macroscopic variables are represented on a discretized grid, or "lattice," and boundary conditions are of the "immersed boundary" type. Furthermore, pressure does not appear explicitly in the Boltzmann equation and incompressible flow is modeled within a low-Mach-number approximation with pressure made proportional to density. Compared with conventional boundary-fitted numerical methods for incompressible flow, the LB method handles complex geometries more easily and is more straightforward to parallelize due to the lack of a pressure term in the Boltzmann equation with corresponding local nature of interactions in physical space.

The LB equation for $f(\mathbf{x}, \xi, t)$ with the Bhatnagar-Gross-Krook (BGK) representation for the collision operator (Bhatnagar, et al..$^{5}$, Chen $\&$ Doolen $^{2}$, Aidun $\&$ Clausen $^{3}$ ) is a form of the Boltzmann equation discretized in velocity space $\boldsymbol{\xi}$ and time t on a spatial lattice discretized in $\mathbf{x}$ :

$$
f_{\alpha}\left(\mathbf{x}+\mathbf{e}_{\alpha} \delta t, t+\delta t\right)-f_{\alpha}(\mathbf{x}, t)=-\frac{1}{\tau}\left[f_{\alpha}(\mathbf{x}, t)-f_{\alpha}^{e q}(\mathbf{x}, t)\right] .
$$

$f_{\alpha}(\mathbf{x}, t)$ is the particle distribution function at location $\mathbf{x}$ and time $t$ discretized in velocity $\mathbf{e}_{\alpha}$, where $\alpha$ is an integer over the number of discretized velocities. The LHS of Eq. (B1) describes the "streaming" of particle distribution functions between a lattice node $\mathbf{x}$ and its neighboring nodes with velocity $\mathbf{e}_{\alpha}$, characterizing bulk exchange of momentum locally in physical space from bulk advection and molecular diffusion. The RHS of Eq. (B1) describes the mixing, or "collision" of molecules that drive the flow locally towards the equilibrium particle distributions $f_{\alpha}^{e q}(\mathbf{x}, t)$. The collision step in the LB algorithm models the diffusion of momentum. with the low Mach number approximation applied to $f_{\alpha}^{e q}(\mathbf{x}, t)$ (below). As is typical, the RHS of Eq. (B1) uses the BGK model ${ }^{5}$ for the collision process with which the distribution functions $f_{\alpha}(\mathbf{x}, t)$ relax towards $f_{\alpha}^{e q}(\mathbf{x}, t)$ at a characteristic diffusion time scale $\tau$ that is related to the fluid viscosity (see Chen \& Doolen ${ }^{2}$ ). Once found, the discretized particle distribution function $f_{\alpha}(\mathbf{x}, t)$ provides the continuum-level velocity and density fields as moments over discretized velocity $\mathbf{e}_{\alpha}$ :

$$
\rho(\mathbf{x}, t)=\sum_{\alpha} f_{\alpha}(\mathbf{x}, t), \quad \rho \mathbf{u}(\mathbf{x}, t)=\sum_{\alpha} f_{\alpha}(\mathbf{x}, t) \mathbf{e}_{\alpha} .
$$

In the LB method spatial and temporal discretization are connected and the lattice must be uniform and velocity discretized to allow "streaming" of particle distributions functions between points defined on the 
lattice at each time step. In 3D the continuum velocity vector $\xi$ can be discretized into 15,19 or 27 components, $\mathbf{e}_{\alpha}^{3,6}$ (referred to as D3Q15, D3Q19 and D3Q27). We applied the D3Q15 approach largely to minimize computational load, with the recognition that our flow structure is relatively simple and our flow Reynolds numbers relatively low, generally order 1 or less. Discussions in the literature ${ }^{7,8}$ suggest that the primary issue with number of discretization components is potential lack of rotational invariance and, correspondingly, incorrect precisions of anisotropic flow structure. These references ${ }^{7,8}$ indicate that these differences only appear at Reynolds numbers two or more orders of magnitude and higher and with high degrees of complexity in the flow topology. It was felt that in the current study the flow topography was sufficiently simple and the Reynolds numbers sufficiently low that the differences in precision among the three methods would be negligible.

For D3Q15, the velocity discretization is given by:

$$
\mathbf{e}_{\alpha}=\left\{\begin{array}{cl}
0, & \alpha=0, \\
( \pm 1,0,0),(0, \pm 1,0),(0,0, \pm 1), & \alpha=1 \text { to } 6 \\
( \pm 1, \pm 1, \pm 1), & \alpha=7 \text { to } 14
\end{array}\right.
$$

The equilibrium distribution function within the low Mach number approximation (Chen \& Doolen²; Qian, et $\left.a l .{ }^{6}\right)$ is given by

$$
f_{\alpha}^{e q}(\mathbf{x}, t)=w_{\alpha} \rho(\mathbf{x}, t)\left[1+3 \frac{\mathbf{e}_{\alpha} \cdot \mathbf{u}}{c^{2}}+\frac{9}{2} \frac{\left(\mathbf{e}_{\alpha} \cdot \mathbf{u}\right)^{2}}{c^{4}}-\frac{3}{2} \frac{(\mathbf{u} \cdot \mathbf{u})^{2}}{c^{2}}\right],
$$

where $w_{\alpha}$ are weighting factors. For D3Q15 these are: $w_{0}=2 / 9, w_{\alpha}=1 / 9$ for $\alpha \sim 1-6$ and $w_{\alpha}=1 / 72$ for $\alpha \sim 7-14 . c=\delta x / \delta t$ is the basic speed on the lattice.

The no-slip boundary condition is most commonly implemented using a first-order "bounce-back" boundary condition applied on a discretized boundary elements that are, by construction, placed half way between the lattice nodes; the particle distribution function representing collections of molecules traveling towards these elements are "bounced back" to the same node, thus creating net zero momentum transport at that boundary element in that direction. Moving boundaries require the addition of motion-generated momentum using an approach first advanced by Ladd ${ }^{9}$. Given that the flows with which we apply the LB approach are typically driven by the motion of the boundaries, higher-order accuracy at the boundaries desirable. We therefore apply a second-order improvement to the bounce-back boundary condition advanced by Lallemand and Luo ${ }^{10}$ that incorporates interpolation to the precise location of the boundary discretized within each lattice cell: if the streaming distance to a solid boundary is less than half the lattice dimension, the particle distribution functions are interpolated before propagation, while if greater than half the lattice dimension, interpolation is conducted after propagation, bounce-back and collision.

\section{Evolution of the Concentration Field}

To evolve the scalar concentration over time within the flow field, we apply the "moment propagation method" developed by Frenkel and Ernst ${ }^{11}$, Lowe and Frenkel ${ }^{12}$, and Merks et al. ${ }^{13}$ with which the scalar concentration $\phi(\mathbf{x}, t)$ is propagated at the continuum level for each scalar using the discretized particle distribution function $f_{\alpha}(\mathbf{x}, t)$ as follows:

$$
\phi(\mathbf{x}, t+\delta t)=\sum_{\alpha} P_{\alpha}\left(\mathbf{x}-\mathbf{e}_{\alpha} \delta t, t+\delta t\right)+\phi(\mathbf{x}, t) \Delta^{*},
$$


where

$$
P_{\alpha}\left(\mathbf{x}-\mathbf{e}_{\alpha} \delta t, t+\delta t\right)=\left[\frac{\hat{f}_{\alpha}\left(\mathbf{x}-\mathbf{e}_{\alpha} \delta t, t_{+}\right)}{\rho\left(\mathbf{x}-\mathbf{e}_{\alpha} \delta t, t\right)}-w_{\alpha} \Delta^{*}\right] \phi\left(\mathbf{x}-\mathbf{e}_{\alpha} \delta t, t\right),
$$

and

$$
\Delta^{*}=1-6 \frac{D_{m}}{c \delta x}
$$

$t^{+}$implies after streaming but before collision. The moment propagation method is limited by an upper bound in Peclet number to maintain numerical stability, which requires

$$
\Delta^{*} \leq \min \left(\frac{f_{\alpha}^{e q}(\vec{u}, \rho)}{f_{\alpha}^{e q}(\vec{u}=0, \rho)}\right) .
$$

The Dirichlet boundary condition $\phi\left(\mathbf{x}_{\text {surface }}, t\right)=C_{S}$ is applied at the particle surface.

\section{Resolution and the Dual Lattice}

As described in the body of the manuscript, to minimize the influence of the boundary on the dissolution process, the relative distances to the boundaries of the computational domain to particle radius were maintained well over an order of magnitude (as large as possible within practical limits): $L / R=25, W / R=$ $H / R=20$ (most cases) and 40 (5 lowest Reynolds number cases), as illustrated in Figure 1. Our aim was high-accuracy prediction of molecular release rate from a drug particle in an unbounded uniform shear flow. Special care was taken to minimize influences of confinement in the vertical on the flow and dissolution process. The domain was sufficiently long in the streamwise direction that steady state was reached before scalar released from the particle surface has reentered the computational domain. Since the concentration field never spread more than a couple diameters in the lateral direction, there was minimal influence of the lateral solid boundaries on the development and evolution of the scalar concentration field. Zero normal flux boundary conditions were applied on the lateral impermeable walls.

To dramatically reduce the computational load from an exceptionally fine uniform grid through the entire computational domain as required by the LB method to resolve exceptionally well a spherical particle in a local shear flow, we applied a dual-lattice method. With this approach a "fine grid" is placed in a subregion surrounding the particle, as shown in Figure 1b, with fine resolution of 44 lattice points over the particle diameter. The method to transfer mass and momentum between the find and coarse grids within the multi-grid strategy was developed by Filippova and Hanel ${ }^{14}$. We apply the improved method by Yu et $a l .{ }^{15}$. Detailed conservation tests by Wang et al. ${ }^{1}$ show that the methods works exceptionally well.

The fine grid occupies a volume extending between approximately 1 to 3 particle radii from the particle in the two lateral directions. Specifically, the fine lattice region was between $h / R$ of 2.1 and 3.6 according to the Reynolds number, with $h / R$ between 3.3 and 3.6 at the smaller Reynolds and Peclet numbers where diffusive effects are strongest, and between 2.1 and 2.6 at the higher Reynolds numbers. In the streamwise direction the fine grid extends to the domain boundaries, as illustrated in Figure 1. Outside this sub-region, the fine lattice couples to a lattice with resolution 6 - 10 times coarser than the fine lattice, depending on Reynolds/Peclet number. 
To demonstrate the quality of the resolution we apply to minimize numerical error, we show in Figure B1 plots of streamwise velocity component along vertical lines in two cases at the extremes in the shear Reynolds number range calculated with the LB method for our analysis and at the highest Schmidt number (Sc) in each case. The gradients near the particle surface are much higher in the high Reynolds/Schmidt number case than in the low Reynolds number case. In both cases the highest gradient regions in velocity and scalar concentration are very well resolved. At the interface between the fine and coarse grids the transition is smooth.
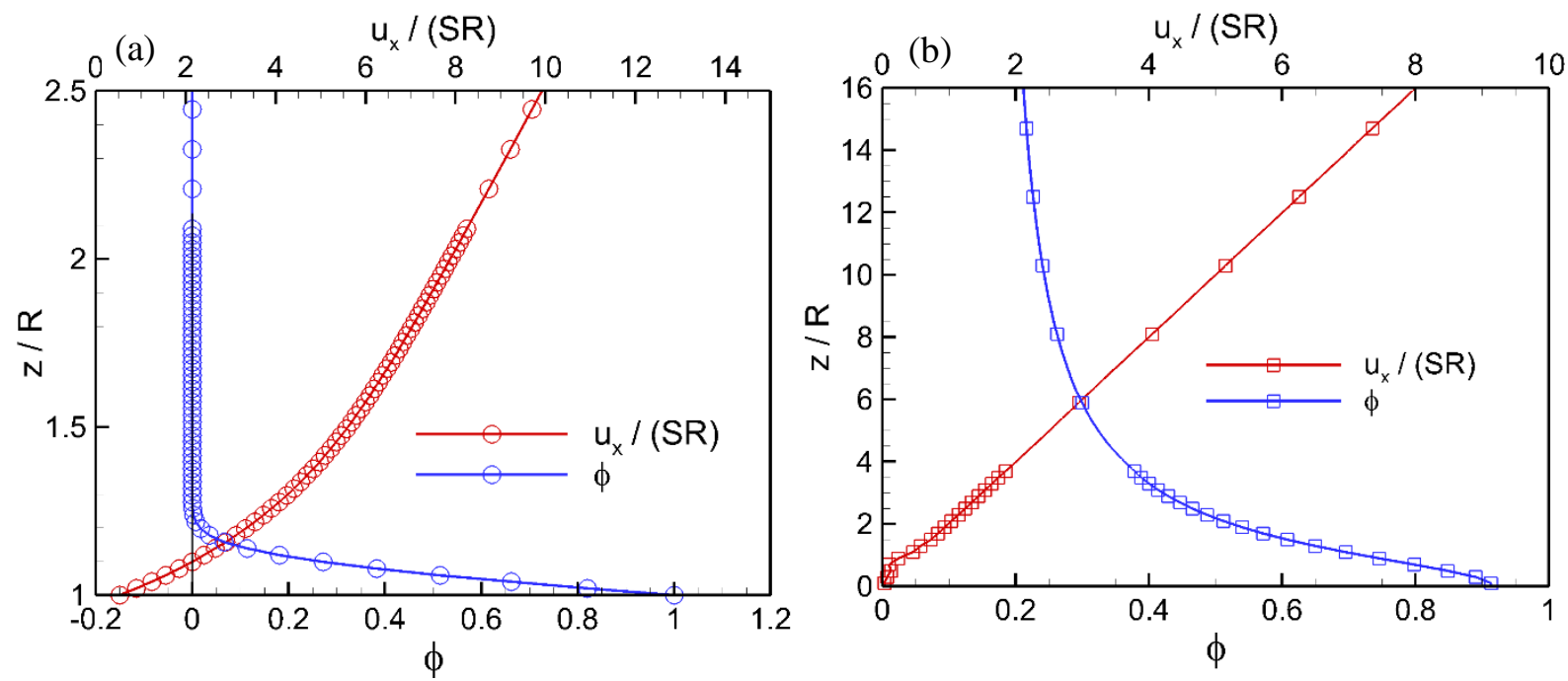

Figure B1. Plots of streamwise velocity and scalar concentration values on lattice nodes along vertical lines (see Figure 1) to demonstrate resolution quality in this study and the transition between the fine and coarse lattices: (a) $\operatorname{Re}_{S}=10, \mathrm{Sc}=40\left(S^{*}=400\right)$, plotted from the top of the spherical particle $(x=0$, $z=R$ ) at each lattice node; (b) $\operatorname{Re}_{S}=10^{-4}, \mathrm{Sc}=10\left(S^{*}=0.001\right)$, plotted from the front of the spherical particle $(x=-R / 2, z=0)$ at each lattice node.

The spherical particle is freely suspended in our Couette flow with spin in response to stress exerted by the fluid on the particle surface expressed as the local exchange of momentum between neighboring "fluid" and "solid" nodes ${ }^{16}$. We evolved the momentum field to steady state before turning on the release of scalar concentration from the particle surface and continued to the steady equilibrium state at which point dissolution rate is calculated at $S^{*}$ and $\operatorname{Re}_{S}$ controlled by specified shear rate, fluid kinematic viscosity and molecular diffusivity. "Steady state" was defined as the time when the normalized rate of change in dissolution rate is below $0.01 \%$ :

$$
\left|\frac{1}{S \dot{Q}} \frac{d \dot{Q}}{d t} \approx \frac{1}{S \dot{Q}(t)} \frac{\dot{Q}(t+\delta t)-\dot{Q}(t)}{\delta t}\right| \leq 10^{-4},
$$

where $\dot{Q}$ is the dissolution rate (mass/time) at time $t$ and $S$ is the flow shear-rate.

\section{Model for Zero Reynolds Number}

For the cases at $\operatorname{Re}_{S}=0$ (Stokes flow) with $S^{*}$ varying from zero to large values, we take advantage of the known analytical solution for velocity surrounding a spherical particle freely-suspended in an infinite simple shear flow ${ }^{17}$, which is given in spherical coordinates as: 


$$
\begin{aligned}
& u_{r}=S r \sin ^{2} \theta \sin \phi \cos \phi\left[1-\frac{5}{2}\left(\frac{R^{3}}{r^{3}}\right)+\frac{3}{2}\left(\frac{R^{5}}{r^{5}}\right)\right] \\
& u_{\theta}=S r \sin \theta \cos \theta \sin \phi \cos \phi\left(1-\frac{R^{5}}{r^{5}}\right) \\
& u_{\phi}=-\frac{S r}{2} \sin \theta+\frac{S r}{2} \sin \theta \frac{R^{3}}{r^{3}}+\frac{S r}{2}(\sin \theta \cos 2 \phi)\left(1-\frac{R^{5}}{r^{5}}\right)
\end{aligned}
$$

where $\theta$ is defined from the $-y$ axis and $\phi$ in the $x$ - $z$ plane (Figures 1,2 in the main manuscript). To develop the most accurate Stokes flow correlation possible between $S h$ and $S^{*}$ within the LB framework, we assign the mass distribution functions values that produce the solution given by Eq. (B10) on which the scalar concentration field evolves using the moment propagation method with fixed surface concentration. More specifically, $\hat{f}_{\alpha}\left(\mathbf{x}-\mathbf{e}_{\alpha} \delta t, t_{+}\right)$in Eq. (B6) is determined for steady state (i.e., equilibrium) by inserting the expressions in Eq. (B10) into the equilibrium distribution function, Eq. (B4) over the computational domain. Because the analytic solution for the particle distribution functions were available, there was no need to provide boundary conditions for velocity. Boundary conditions were only required for concentration, $\phi$. This approach both maximizes accuracy and greatly reduces computational cost.

\section{References for Appendix $B$}

1. Wang Y, Brasseur JG, Banco GG, Webb AG, Ailiani AC, Neuberger T. Development of a latticeBoltzmann method for multiscale transport and absorption with application to intestinal function. Computational Modeling in Biomechanics. Springer, 2010.

2. Chen S, Doolen GD. Lattice Boltzmann method for fluid flows. Ann. Rev. Fluid Mech. 1998; 30: 329364.

3. Aidun CK, Clausen JR, Lattice-Boltzmann method for complex flows. Ann. Rev. Fluid Mech. 2010; 42: 439-472.

4. Shan X, Yuan X, Chen H. Kinetic theory representation of hydrodynamics: a way beyond the NavierStokes equations. J. Fluid Mech. 2006; 550: 413-441.

5. Bhatnagar PL, Gross EP, Krook M. A model for collision processes in gases. I: small amplitude processes in charged and neutral one-component system. Phys. Rev. 1954; 94: 511-525.

6. Qian Y, d'Humieres D, Lallemand P. Lattice BGK methods for Navier-Stokes equation. Europhys. Lett. 1992; 17: 479-484.

7. Nash RW, Carver HB, Bernabeu MO, Hetherington J, Groen D, Krüger T, Coveney PV, Choice of boundary condition for lattice-Boltzmann simulation of moderate-Reynolds number flow in complex domains. PRE. 2014; 89: 023303.

8. White A, Chong C, Rotational invariance in the three-dimensional lattice Boltzmann method is dependent on the choice of lattice. J. Comput. Physics. 2011; 230: 6367-6378.

9. Ladd AJC. Numerical simulations of particulate suspensions via a discretized Boltzmann equation. Part 1. Theoretical foundation. J. Fluid Mech. 1994; 271: 285-309.

10. Lallemand P, Luo L. Lattice Boltzmann method for moving boundaries. J. Comput. Phys. 2003; 184 : 406-421. 
11. Frenkel D, Ernst MH. Simulation of diffusion in a two-dimesional lattice-gas cellular automation: a set of mode-coupling theory. Phys. Rev. Lett. 1989; 56: 2165-2168.

12. Lowe CP, Frenkel D. The super long-time decay of velocity fluctuations in a two dimensional fluid. Physica A. 1995; 220: 251-260.

13. Merks RMH, Hoekstra AG, Sloot PMA. The momentum propagation method for advection-diffusion in the lattice Boltzmann method: validation and Peclet number limit. J. Comput. Phys. 2002; 183: 563576.

14. Filippova O, Hanel D. Grid refinement for lattice-BGK models. J. Comput. Phys. 1998; 147: 219-228.

15. Yu D, Mei R, Luo L, Shyy W. A multi grid lattice Boltzmann method for viscous fluid flows. Int. J. Num. Meth. Fluids. 2002; 39: 99-120.

16. Bouzidi M, Firdaouss M, Lallemand P, Momentum transfer of a Boltzmann-lattice fluid with boundaries. Phys. Fluids. 2001; 13: 3452-3459.

17. Leal LG, Advanced Transport Phenomena - Fluid Mechanics and Convective Transport Processes. Cambridge University Press, 2007. 


\section{APPENDIX C: \\ Sensitivity and Application}

As was discussed in the manuscript in context with Figures 5 and 10, sensitivity in the response of Sherwood number to changes in Peclet and Reynolds number depends on the $S^{*}$ and $\operatorname{Re}_{S}$ regime, with greatest sensitivity to changes in $S^{*}$ and $\operatorname{Re}_{S}$ tending to occur in the lower and higher $S^{*}$ ranges, respectively. We quantify these sensitivities in Figures A1 (a) and (b) through the derivatives of $S h$ with respect to $S^{*}$ and $\operatorname{Re}_{S}$ which, using Eqs. (14) and (15) in the manuscript, are given by,

\section{Correlation 1:}

$$
\begin{gathered}
\frac{\partial(S h)}{\partial S^{*}}=\frac{\partial\left(S h_{0}\right)}{\partial S^{*}}+e^{a} \operatorname{Re}_{S}^{c} \frac{b}{\left(S^{*}\right)^{1-b}}, \\
\frac{\partial(S h)}{\partial \operatorname{Re}_{S}}=e^{a}\left(S^{*}\right)^{b} \frac{c}{\operatorname{Re}_{S}^{1-c}},
\end{gathered}
$$

Correlation 2:

$$
\begin{gathered}
\frac{\partial(S h)}{\partial S^{*}}=\frac{\partial\left(S h_{0}\right)}{\partial S^{*}}+e^{a} \operatorname{Re}_{S}^{c}\left(b+d \ln \operatorname{Re}_{S}\right) \frac{1}{\left(S^{*}\right)^{1-\left(b+d \ln \operatorname{Re}_{S}\right)}} \\
\frac{\partial(S h)}{\partial \operatorname{Re}_{S}}=e^{a}\left(S^{*}\right)^{b}\left(c+d \ln S^{*}\right) \frac{1}{\operatorname{Re}_{S}^{1-\left(c+d \ln S^{*}\right)}}
\end{gathered}
$$

where the coefficients are given in Table 1 and the function $S_{0}\left(S^{*}\right)$ is given by Eqs. (9) in the manuscript. Isocontour plots of $\partial(S h) / \partial S^{*}$ and $\partial(S h) / \partial \operatorname{Re}_{S}$ are shown in Figures $\mathrm{C} 1(\mathrm{a})$ and (b). Equations (C1) (C4) indicate the existence of singularities in the sensitivity to $S^{*}$ at $S^{*}=0$ and the sensitivity to $\operatorname{Re}_{S}$ at $\operatorname{Re}_{S}=0$. Thus we anticipate strong sensitivities in these two limits.

Using the red-yellow isocontours as an indicator of "highest" sensitivity, Figure C1(a) shows that the highest sensitivity to changes in $S^{*}$ occur roughly in the range $S^{*} \sim 0$ to 20 at all $\operatorname{Re}_{S}$, with an apparent singularity at $S^{*}=0$. The range in $S^{*}$ of high sensitivity increases gradually with increasing $\operatorname{Re}_{S}$. This is a significant result because the release of molecules from the surfaces of drug particles tends to occur at lower $S^{*}$ with $\operatorname{Re}_{S}$ approaching zero. The high sensitivity to changes in $S^{*}$ degrades rapidly with increasing $S^{*}$ to small values at $S^{*}$ exceeding roughly 200-300 at Reynolds numbers below $\sim 1$. However at these high $S^{*}$ values, the sensitivity to $S^{*}$ increases with increasing $\operatorname{Re}_{S} \gtrsim 1$, especially at $S^{*}$ exceeding $\sim 400-500$.

Again using the red/yellow isocontours as a marker of highest sensitivity, Figure C1(b) indicates that the highest sensitivity to changes in $\operatorname{Re}_{S}$ occurs at very low $\operatorname{Re}_{S}$ (relative to 1) with an apparent singularity at $\operatorname{Re}_{S}=0$. The range in $\operatorname{Re}_{S}$ where Sherwood number is highly sensitive to changes in $\operatorname{Re}_{S}$ increases with increasing $S^{*}$, with high sensitivity particularly apparent at small $\operatorname{Re}_{S}$ when $S^{*}$ exceeds roughly 100 . Conversely, sensitivity to changes in $\operatorname{Re}_{S}$ is very low at $S^{*}$ below roughly 10 . Sensitivity drops rapidly with increasing $\operatorname{Re}_{S}$ especially at lower $S^{*}$. At $S^{*}$ below $\sim 10$, there is negligible sensitivity to changes in $\operatorname{Re}_{S}$

From an applications perspective, one might ask over what range of parameters the Stokes-flow correlations of Eq. (9) in the manuscript could be applied with good accuracy. We address this question in Figure C1 (b) and (c) where we plot the relative deviation of $S h$ from $S h_{0}$ : 


$$
\Delta\left(S^{*}, \operatorname{Re}_{S}\right)=\frac{S h-S h_{0}}{S h_{0}} .
$$

Using 10\% deviation as demarcation of "reasonable" accuracy, Figure 1(c) shows that reasonable accuracy is obtained using the Stokes flow correlation at "small" $S^{*}$, at "small" $\operatorname{Re}_{S}$ and especially in a broader range of $S^{*} \leqslant 50$ when $\operatorname{Re}_{S} \leqslant 1$. Given that $\operatorname{Re}_{S}$ rarely, if ever, approaches 1 for typical drug particle dissolution (see Figure 5), in Figure C1(d) we expand Figure C1(c) for the region $\operatorname{Re}_{S} \leq 1$. We observe that the Stokes flow model is reasonable (within 10\%) over the entire $S^{*} \sim 0-500$ range when $\operatorname{Re}_{S}<0.01$ (unlikely at $S^{*} \approx 500$, see Figure 5 in the manuscript), but is reasonable for $S^{*} \lesssim 100$ when $\operatorname{Re}_{S} \leqslant 0.5$, which is quite likely.
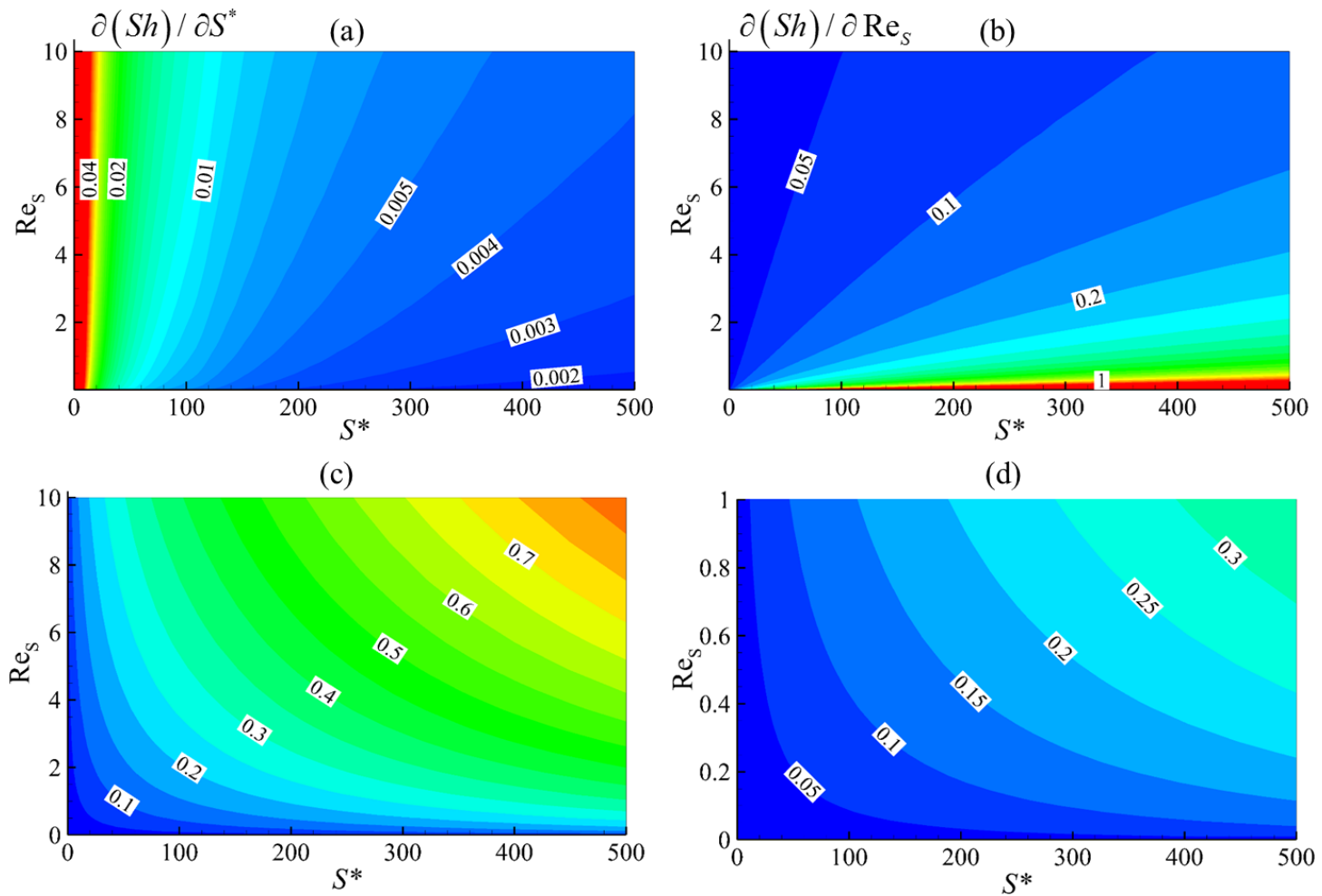

Figure C1. (a),(b) Isocontours of the derivative of Sh with respect (a) $S^{*}$ and (b) $\operatorname{Re}_{S}$ as indicators of sensitivity in Sh to changes in $S^{*}$ and Res. (c),(d): Isocontours of the relative deviation $\Delta$ in Sherwood number from the Stokes flow correlation, Eq. (19) in the manuscript. Fig. (d) is an expanded version of (c) at $\operatorname{Re}_{S}<1$, typical of dissolution form drug particles and where differences from the Stokes flow correlation are relatively minor. In all figures the more accurate correlation 2 was used. 